\title{
Exploration and Evaluation of AR, MPCA and KL Anomaly Detection Techniques to Embankment Dam Piezometer Data
}

\author{
In-Soo Jung ${ }^{1}$, Mario Berges ${ }^{2}$, James H. Garrett Jr${ }^{3}$, Barnabas Poczos ${ }^{4}$ \\ ${ }^{1}$ Ph.D. Student, Dept. of Civil and Environmental Engineering, Carnegie Mellon University, \\ 5000 Forbes Avenue, Pittsburgh, PA 15213. E-mail: insoojungs@ gmail.com \\ ${ }^{2}$ Assistant Professor, Dept. of Civil and Environmental Engineering, Carnegie Mellon \\ University, 5000 Forbes Avenue, Pittsburgh, PA 15213. E-mail: mberges@ andrew.cmu.edu \\ ${ }^{3}$ Dean of Carnegie Institute of Technology, Carnegie Mellon University, 5000 Forbes Avenue, \\ Pittsburgh, PA 15213. E-mail: garrett@cmu.edu \\ ${ }^{4}$ Assistant Professor, Machine Learning Department, Carnegie Mellon University, 5000 Forbes \\ Avenue, Pittsburgh, PA 15213. E-mail: bapoczos@cs.cmu.edu
}

\begin{abstract}
In the U.S., the current practice of analyzing the structural integrity of embankment dams relies primarily on manual a posteriori analysis of instrument data by engineers, leaving much room for improvement through the application of advanced data analysis techniques. In this research, different types of anomaly detection techniques are examined in an effort to propose which data analytics are appropriate for various anomaly scenarios as well as piezometer locations. Moreover, both the parametric (Auto Regressive [AR] and Moving Principal Component Analysis [MPCA]) and nonparametric (Kullback-Leibler Divergence [KL]) techniques are applied in order to test if the widely-held assumptions about piezometer data, i.e., linearity between piezometer data and pool levels, as well as normally distributed piezometer data, are necessary in the anomaly detection task. In general, KL performs better than MPCA and AR, and delivers more consistent results throughout the different piezometers and anomaly scenarios. Given that $\mathrm{KL}$ is a nonparametric technique, the authors conclude that the prior assumptions about piezometer data do not always provide the best performance for anomaly prediction.
\end{abstract}

Keywords: structural health monitoring, dam safety, anomaly detection, statistical techniques

\section{Introduction}

There are 84,000 dams in the U.S. with an average age of 53 years. Since the design life for most of these dams is 50 years, this means they are in need of extensive rehabilitation [1]. Moreover, dams have received a grade of D in the 2013 ASCE infrastructure report card [2]. While dams provide drinking water, hydroelectric power, flood control, recreation and many other benefits, dams can pose significant risks to people living around them. According to ASDSO and FEMA [3], even though dam failure is a low probability event, it poses a high risk to the population around the area. Moreover, the probabilities are still high enough that failures typically happen 

the U.S. infrastructure systems that needs to be improved. To efficiently expedite the improvement, systematic inspections as well as more advanced monitoring systems are necessary.

Embankment dams are the most common type of dam in use today [3]. In general, embankment dams are constructed of natural earthen materials, usually soil and rock [4]. In embankment dams, water seeps through different soil layers of the dam, and any changes in this behavior may signal problems. One of the leading causes of failure of embankment dams has been internal erosion, which can occur due to normal operations that may pose higher risks to a dam than remote loading conditions such as floods and earthquakes [5]. Internal erosion problems are usually detected by periodic visual inspection and seepage measurement [6]. However, since they mostly occur without any visual signs, it is often too late by the time problems are actually identified. Any anomalous changes in pore pressure within a dam signal potential seepage problem. Thus, monitoring pore pressure as well as water levels of an embankment dam to observe potential seepage problems is important; anomalies that are indicative of internal erosion problems should be detected in advance to prevent catastrophic consequences.

Engineers monitor a variety of data collected from different instruments regularly to ensure that a dam is performing properly and as expected [7]. If there is any abnormal change in flow rates or volumes of seepage over time, for example, this can be an indication of various problems, such as piping (pathways of water flow through the soil), cracks, malfunctions of the piezometers, etc. However, interpreting such abnormal changes or fluctuations in measurements from the instruments is not always straightforward. Embankment dams, like most other civil infrastructure systems, are exposed to unpredictable environments. Moreover, their design specifications and as-is properties are not generally known due to, among other things, their age and the difficulties associated with assessing their internal structure. Hence, accurately evaluating measurements collected from instruments used to monitor dam behavior is not an easy task, requiring sound engineering judgment and analysis, as well as robust statistical analysis techniques to prevent misinterpretation. In the U.S., the current practice of analyzing the structural integrity of embankment dams relies primarily on manual a posteriori analysis of instrument data by engineers, providing an opportunity for improvement.

Piezometers are the most commonly used instruments to monitor the water level in dams, which can often be used to compute pore water pressure [8]. Besides the reservoir pool, piezometers are influenced by many factors such as precipitation, tailwater present in downstream, soil permeability, temperature, etc. For embankment dams, piezometer levels are highly influenced by reservoir levels, so they are usually compared together to monitor the seepage as well as to check the condition of the piezometers. In addition to regularly collected piezometric and reservoir readings, the data collected during past historical events, such as flood events, high pool operation, etc., are also considered so that the dam behavior during such extreme events can be understood. Engineers look for any anomalies by observing correlation plots that graph unexpected deviations from the established norm (or the trend line) that reflects the relationship between the two variables (see Figure 1). By observing how much the collected data are tracing 

as expected or not. However, it is often difficult to quantitatively determine if identified deviations depicted in the correlation plot actually represent problematic behaviors. In addition, besides yearly checkups, more intense analysis of the collected instrument data, including piezometer data, is normally done in 5+-year intervals in the U.S. Since dams often have slow responses, relatively small anomalies, which could be an initiation of a catastrophic event, may be overlooked. The traditional practice that subjectively detects deviations from the historical readings using time series or correlation plots of raw data may not be accurate enough to capture anomalous changes over time. Thus, a more advanced way of analyzing collected instrument data is necessary to improve interpretation of the data and thereby enhance dam safety.

Given that different parts of a dam behave differently and undergo complicated processes over time, it is possible to improve the traditional anomaly detection approach using more advanced data analysis techniques. Thus, in this paper, different types of anomaly detection techniques are presented in an effort to propose/recommend data analytics that are more appropriate for various anomaly scenarios as well as piezometer locations. In this work, the authors test Auto Regressive (AR) model, Moving Principal Component Analysis (MPCA) and Kullback-Leibler divergence, which entail different characteristics.

This paper is organized as follows. In Section 2, we briefly introduce embankment dams and the mechanisms of internal erosion in an effort to emphasize the importance of detecting internal erosion problems in advance. In Section 3, we introduce a case study dam on which our analyses are based. We also describe the details of the simulations performed for the various scenarios considered in this work. Section 4 presents three anomaly detection techniques that are evaluated as part of this research. We also describe the principles and characteristics of those techniques as well as why they are appropriate for the anomaly detection task given our data. In Section 5, we describe the experiments we conduct, and the corresponding results are presented in Section 6. Finally, Section 7 presents a discussion of these results and the conclusions.

\section{Internal Erosion}

According to Torres [9], internal erosion in embankment dams is defined as "a process that develops a concentration of seepage flows and in time may develop large cavities that may produce embankment failure with a catastrophic and uncontrolled release of the reservoir." The mechanisms of internal erosion are complex especially given that various flaws and physical factors can lead to internal erosion. Classical piping, progressive erosion, blowout, and scour are the well-known mechanisms of internal erosion. Internal erosion can happen due to, but not limited to, improper filters; pressure relief measures not being used at seepage discharge points; soil not properly compacted adjacent to outlet pipes; sharp changes in foundation grades or slopes; water flows along cracks or other defects in the soil; pressurized water flows into the embankment; etc. In most cases, soil particles are initially removed at the discharge point of the seepage, and pipes in the soil tend to enlarge and progress upstream with increasing flow quantity [10]. 

shown in Figure 2, which shows the specific case of internal erosion in embankment initiated by concentrated leak [11].

As explained above, internal erosion is a complicated process, and site-specific conditions should be also considered to better understand its likelihood to develop further. In addition, an internal erosion problem cannot be completely analyzed using only numerical models; additional information on dam and soil behavior needs to be assessed to determine the risks associated with internal erosion [12]. Any abnormalities, such as heave, cracks, removal of materials, etc. in a dam may or may not continue to develop actual internal erosion. However, such observations should be carefully examined due to the possibility of being further developed to initiate internal erosion. Thus, it is difficult to state that detected anomalies from instrumentation readings and/or visual observations are actual internal erosion; rather, they should be considered as potential indicators of internal erosion. In some situations, problems can be self-healed or not progress further based on various factors. Thus, secondary consequences of internal erosion are not considered in this work, meaning that the authors do not judge whether detected anomalies will always lead to internal erosion problems, but rather, try to detect any types of potential anomalous responses that may possibly continue to real internal erosion.

Our simulated piezometer signals correspond to an ideal condition where the changes seen in the response of the piezometer to varying reservoir levels are solely the effect of a change in the hydraulic conductivity of sections of the dam. Responses from real piezometers could undergo similar changes for various reasons other than the ones simulated, which would increase the false alarm rate of our approach. However, given that domain experts prioritize sensitivity over specificity, and that simulating other phenomena that may result in similar response changes is difficult, we believe that the results shown in the paper are informative and can be used as a baseline for future work on improving the specificity of the test under a wider set of anomalies.

\section{Data Collected from Simulation}

Since the anomalous problems often occur inside embankment dams without visual signs until they become severe enough to be observed, it is hard to know exactly when such problems are initiated. Thus, a simulation process is necessary in order to accurately evaluate the ability of the anomaly detection techniques to detect anomalies long before they become severe enough to be visually observed. Moreover, such simulations will feed additional information, such as how piezometric levels would be affected by the specific physical processes being simulated, which have been hard for engineers to understand based only on observations and data collected from real embankment dams. The datasets collected from these embankment dam simulations will not only benefit the validation of anomaly detection approaches, but also contribute to the understanding by dam engineers of the behavior of piezometers in embankment dams under different conditions. 


\subsection{Case Study Dam}

In order to simulate realistic anomalous embankment dam scenarios, a case study dam located in the eastern part of the U.S. was used because its reservoir and instrumentation measurements were accessible to the research team. The dam is an earth and rock filled structure and is composed of a central core of impervious rolled fill with the upstream side slope protected by rock fill on gravel bedding. On the downstream of the core, there is a large zone of rock fill. The dam was built in 1961, and most of the instrumentation systems had been manually collected and evaluated. After installing a new piezometer instrument system in 2004, the data collection has been automated. Now, there are a total of 26 piezometers installed, and starting from 2009, measurements are collected every 4 hours. In our work, in order to analyze the piezometer data in a consistent frequency for a longer period of time, we used the data collected since 2006 and resampled them to have the same frequency, i.e., every 24 hours.

\subsection{Anomalous Data Collection}

Recently, numerical analyses coded into computer programs have been the most widely used method to analyze seepage issues. Specifically, 2-dimensional (2D) or 3D numerical models are often developed to analyze seepage issues [13]. Such models are often used for simulation of infiltration, seepage and seepage path, etc., so that the performance of the dam can be validated. In this work, SEEP/W was used to model a section of the case study dam in order to simulate various anomaly scenarios, and consequently, to collect corresponding piezometer data. SEEP/W is a finite element software program for analyzing ground water and excess pore-water pressure dissipation problems in a porous media. Using SEEP/W, it is possible to vary relevant parameters, such as reducing the hydraulic conductivity of soil layers and/or the core, thus obtaining datasets that correspond to different phases of internal erosion. In addition, since SEEP/W adopts an implicit numerical solution to solve Darcy's equation, users can perform both steady-state and transient seepage analysis during specified time sequences.

The existing 26 piezometers already installed in the case study dam are clustered in the core area, so some of the existing piezometers and several new locations were selected to collect piezometer data that have various response characteristics to the pool levels, and are spatially spread along the dam section. For all transient analyses, users need to adjust time steps appropriately in order to obtain computed results accurately. It should not be too fast or too slow. After setting geometry, meshing, soil materials, soil properties, and boundary conditions based on our case study dam, we performed the transient analyses using the daily reservoir levels obtained from the case study dam as the inputs. Given the daily reservoir levels for five and a half years, specifically Mar. 2006 to Sep. 2011, corresponding daily piezometer data were calculated from the 10 specific locations in the model as shown in Figure 3. Table 1 provides more details about each piezometer and the corresponding dam properties.

In this work, the obtained piezometer datasets from the case study dam under the normal condition became the baseline data. In order to see how reliable SEEP/W is, we compared the 
calculated piezometer data to the field piezometer data collected at PZ 4 location for the period of 2008 days being studied. The dam model was simulated using the actual reservoir levels as the forcing function. The mean value of the simulated piezometer data was 822.0639 while the mean value of the field piezometer data was 822.0650. The correlation coefficient and the coefficient of determination (R-square) between the two time series were 0.9980 and 0.9961 , respectively. Similar results were obtained at the other piezometer locations. These comparison results show that the SEEP/W model is able to accurately simulate our case study dam. In the following section, we will demonstrate how we obtained the anomaly datasets under various anomalous conditions by varying properties of the soil materials.

\subsection{Anomaly Scenarios}

Out of many different internal erosion mechanisms possible, the authors only considered two that can be simulated using SEEP/W and possibly occur given the properties and history of the case study dam.

\section{Case 1: Foundation erosion}

In most cases, internal erosion occurs from the toe area and develops backwards towards an embankment. This is often caused by the constant migration of soil particles towards free exits or into coarse openings [14]. Once piping initiates and eventually develops further, the hydraulic conductivity of affected soil materials change due to such soil erosion. Such backward erosion piping can occur from either the foundation or embankment. In this work, anomalies in the downstream foundation of the case study dam are presented, whose soil property is designated as river deposit.

\section{Case 2: Pervious layer in core}

Another anomaly scenario is internal erosion caused by poorly compacted zones (or high permeability zones) within an embankment core. When constructing an embankment dam, each soil layer is compacted on top of the previously compacted layers. However, due to occasional freezing on seasonal shutdown, poor compaction, and many other reasons, highly permeable zones/ layers can be possibly formed within a core, foundation or even at abutment contacts. In this work, we simulated a pervious layer in the core.

In order to generate various anomaly scenarios over the two internal erosion cases, we varied severities and durations of the anomaly scenarios as follows:

- Severity Low: original hydraulic conductivity times 2, 4, 6, 8, 10

- Severity High: original hydraulic conductivity times 10, 25, 50, 75, 100

- Severity extremely-High: original hydraulic conductivity times $10^{1} 10^{2}, 10^{3}, 10^{4}$

- Duration Slow: three months for each change 
- Duration Fast: one month for each change

- Uniformity Graded: the severities of the anomalies are varied in the direction of anomaly progression along the anomalous area/ layer

- Uniformity Uniform: the severities of the anomalies are uniformly distributed along the anomalous area/layer

Thus, as summarized in Table 2, the combinations of the severity, duration and uniformity generated a total of 10 anomaly scenarios, in which the first eight scenarios correspond to the foundation erosion case while the last two scenarios correspond to the pervious core case.

For the first anomaly mechanism, i.e., foundation erosion in river deposit, we created a thin $1 \mathrm{ft}$. thick and $340 \mathrm{ft}$. long layer within the river deposit as indicated with the red line in Figure 3. Figure 4 shows the zoom-in version of this red thin layer of Figure 3. We then divided the layer into five equal segments in which various anomalies could be introduced. Each segment was assigned with various hydraulic conductivity values over various durations. In order to simulate the backwards erosion, which often starts from the toe area, we applied the anomaly scenario of 'Uniformity: Graded'. Here, the hydraulic conductivity of the right most segment, or the $5^{\text {th }}$ segment, was increased, first.

For example, in the case of Scenario 1 ('Severity: Low, Duration: Slow, and Uniformity: Graded'), the hydraulic conductivity of the $5^{\text {th }}$ segment of the layer was increased by 2 times the original hydraulic conductivity of the river deposit for three months. Then in the next three months, the hydraulic conductivity of the $4^{\text {th }}$ segment was increased by 2 times and that of the $5^{\text {th }}$ segment by 4 times, and so on. Figure 5 illustrates how we increased the hydraulic conductivity of each segment over time in the case of Scenario 1. This particular anomaly scenario spans a total 15 months (3 months times 5 segments). In the 'Uniformity: Uniform' case, the hydraulic conductivities of all five segments were uniformly increased. That is, the hydraulic conductivities of all five segments are the same for three months or one month depending on Duration: Slow or Fast, respectively.

To simulate a pervious core layer, another thin layer was created along the core as indicated with the green line in Figure 3. Since the core had been compacted to be impervious, its hydraulic conductivity was increased more severely, i.e., $10^{1} 10^{2}, 10^{3}, 10^{4}$ times the original magnitude ('Severity: extremely-High'), and applied only a 'Uniformity: Uniform' scenario to simulate a pervious layer. Scenario 9 and 10 in Table 2 correspond to this poorly compacted pervious layer in the core area.

Figure 6 shows the time series of one of the piezometers (PZ 4) based on various scenarios. The figure shows the data from Jan. 2009 to Apr. 2010 only to highlight the nature of the anomalous data in each scenario. It is shown that PZ 4 responds differently in the different scenarios.

Figure 7 shows the time series of three different piezometers that were generated from Scenario 1. Even though the data were generated from the same anomaly scenario, it is clear that different 

while some also involve trend changes, probably due to time lags caused by changes in seepage rates. In Figure 7, PZ 3 has a different response trend compared to PZ 2 and PZ 10. Also, while PZ 2 and PZ 10 have similar trends in their responses, PZ 2 readings have more rapid variations, and PZ 10 readings are a smoother version of PZ 2. PZ 2 and PZ 10 are installed in the same soil material, but PZ 2 is located close to the reservoir level as one of the upstream piezometers. Thus, PZ 2 tends to be more responsive to the pool level changes and has higher frequencies in its responses.

\section{Anomaly Detection Techniques}

The objective for the research described in this paper is to examine several data analysis techniques to detect anomalous patterns in piezometer data that do not follow its previous historical behavior or do not behave as expected. Since the $19^{\text {th }}$ century, anomaly detection has been widely studied by the statistics community, and there have been a variety of anomaly detection techniques developed for certain application domains and for more generic problems [15]. Anomaly detection techniques/algorithms should be selected by considering whether they are suitable for the data sets in terms of the correct distribution model, the correct attribute types, the scalability, the required running speed, the availability and accuracy of the labels, etc. [16]. In addition, selecting appropriate approaches depends on the data type, availability of data labels, how one wishes to detect anomalies and to handle them [16]. In this paper, the authors carefully investigate how an anomaly should be defined in piezometer data analysis as well as the sensitivity required and the validation methods that should be used.

Piezometer data are collected at a regular frequency (e.g., every 4 hours, 12 hours, etc.), so temporal information should not be ignored but perceived as an important factor. Moreover, since piezometer data are highly affected by pool levels, seasonality present in data should be also considered. As mentioned above, anomalies are defined as data or patterns that deviate from the normal data. Assuming that the first few years of data are normal, anomalies are detected by looking for any unexpected changes in that data over time.

Based on the nature of the piezometer data, three types of anomaly detection techniques were selected to be studied as part of our research. The three techniques evaluated were: Auto Regression (AR), Moving Principal Component Analysis (MPCA), and Kullback-Leibler (KL) Divergence, which will be explained in detail below. These three techniques can handle multidimensional data (pool and piezometer data) and be adjusted to retain temporal information. In addition, since they entail different characteristics, it would be possible to provide relevant insights to various anomaly scenarios as well as different piezometers. Even though AR is one of the most widely used time series techniques, there is a limitation in such a predictive technique, because accurately modeling time series with some noise present is challenging especially given that AR only considers the linear dependence in a time series [17]. Thus, techniques that initially extract features from the data (e.g., MPCA and KL) were also tested, which are more robust to noise present in the data. 
Given that piezometer data fluctuate based on pool levels, one of the most common assumptions made by practitioners is that the two should be linearly related under ideal conditions. Moreover, especially when looking for any deviations from normal data, engineers tend to assume that the data come from normal (Gaussian) distribution, which is the simplest mathematical model that can be used. However, based on where piezometers are located along the dam, their measurements can be either linear/nonlinear or highly correlated/uncorrelated in response to pool levels. This means that detecting any deviations with a Gaussian approach may not be appropriate. Thus, the research test both parametric and nonparametric techniques, MPCA and KL divergence, respectively, to see if the existing assumptions on linearity as well as Gaussian distribution are appropriate when analyzing piezometer data. In the following sections, each model and technique are explained in more detail.

\subsection{Autoregressive (AR) Model}

One of the most widely used stochastic time series models is Autoregressive Integrated Moving Average (ARIMA) [18,19]. While ARIMA models have been mainly adopted in economic and statistics domains, they also have been popular for structural health monitoring or damage detection [20, 21, 22]. ARIMA assumes that a time series dataset follows a normal distribution and has a linear form meaning that future values of the time series are estimated based on linear combination of previous values of the series. An ARIMA model can be divided into subclasses, such as Autoregressive (AR), Moving Average (MA), and Autoregressive Moving Average (ARMA) models [19]. Particularly, the AR model is expressed in terms of past values, MA model is expressed in term of past errors, and ARMA is a combination of AR and MA models.

In order to determine the most appropriate model given time series data, autocorrelation (ACF) and partial autocorrelation functions (PACF) should be computed. Autocorrelation measures the linear dependence of a variable at different lags while partial autocorrelation measures the linear dependence between different lags after removing the mutual autocorrelation in-between the lags (controls intermediate autocorrelations). Eq. (1) and Eq. (2) represent the ACF and PACF of a time series, $x_{t}$, at lag $j$, respectively [23].

$$
\begin{gathered}
\rho_{j}=\operatorname{corr}\left(x_{t}, x_{t-j}\right)=\frac{\operatorname{cov}\left(x_{t}, x_{t-j}\right)}{\sqrt{\operatorname{var}\left(x_{t}\right) \operatorname{var}\left(x_{t-j}\right)}} \\
\phi_{j j}=\operatorname{corr}\left(x_{t+j}-\hat{x}_{t+j}, x_{t}-\hat{x}_{t}\right), j \geq 2
\end{gathered}
$$

where $\hat{x}_{t+j}$ denotes the regression of $x_{t+j}$ on $\left\{x_{t+j-1}, x_{t+j-2, \cdots}, x_{t+1}\right\}$.

After examining these functions, either AR or MA is selected to appropriately model time series. For example, if a PACF plot of a time series displays a sharp cutoff at a certain lag while ACF decays more slowly, the time series are said to have an AR signature given that AR considers 
cumulative effects of all previous values. In this case, the order number is determined based on the point at which lag PACF cuts off. The opposite scenario applies for a MA model. In MA models, since only errors at certain lags (let's say, $p$ ) affect the current value, ACF will cutoff after lag $p$ while PACF will decay more slowly. All of the piezometers analyzed in this paper have AR signatures as shown in Figure 8; each ACF decays slowly while PACF cuts off sharply at a certain lag. So, this paper focuses on developing AR models.

Eq. (3) below shows an AR model of order $n$. The $x_{t}$ represents the value of $x$ at time, $t$. The equation shows that $x_{t}$ can be estimated using a linear relationship among $n$ previous $x$ values and their corresponding coefficients, $a$ 's. $\varepsilon_{t}$ is the Gaussian white noise term. There are different ways of estimating such coefficients, e.g., least-squares, Yule-Walker approach [24], Forwardbackward approach [25, 26], etc.

$$
x_{t}=\left(a_{1} x_{(t-1)}+a_{2} x_{(t-2)}+a_{3} x_{(t-3)}+\cdots+a_{n} x_{(t-n)}\right)+\varepsilon_{t}
$$

\subsubsection{Vector Autoregressive (VAR) Model}

Eq. (3) is based on a univariate time series. However, since piezometer readings are highly affected by the pool levels, the relationship between the pool and piezometer readings should be considered together. Vector Auto Regressive model (VAR) is a multivariate time series model that can track many series at once instead of a single time series at a time. Since VAR jointly considers dynamics among multiple time series, it is possible to recognize how much one variable is influenced by the other variables. Eq. (4) shows a VAR $(n)$ model:

$$
\left(\begin{array}{c}
x_{1, t} \\
\vdots \\
x_{d, t}
\end{array}\right)=\left(\begin{array}{c}
c_{1} \\
\vdots \\
c_{d}
\end{array}\right)+\left(\begin{array}{ccc}
a_{1,1}^{1} & \cdots & a_{1, n}^{1} \\
\vdots & \ddots & \vdots \\
a_{d, 1}^{1} & \cdots & a_{d, n}^{1}
\end{array}\right)\left(\begin{array}{c}
x_{1, t-1} \\
\vdots \\
x_{d, t-1}
\end{array}\right)+\cdots+\left(\begin{array}{ccc}
a_{1,1}^{n} & \cdots & a_{1, n}^{n} \\
\vdots & \ddots & \vdots \\
a_{d, 1}^{n} & \cdots & a_{d, n}^{n}
\end{array}\right)\left(\begin{array}{c}
x_{1, t-n} \\
\vdots \\
x_{d, t-n}
\end{array}\right)+\left(\begin{array}{c}
\varepsilon_{1, t} \\
\vdots \\
\varepsilon_{d, t}
\end{array}\right)
$$

where $x_{d, t}$ denotes $d^{\text {th }}$ time series at a time $t, a^{n}$ 's are AR coefficients that correspond to $n^{\text {th }}$ lag, and $\varepsilon_{t}$ is a vector of uncorrelated errors.

\subsection{Moving Principal Component Analysis}

By linearly decomposing a data matrix into a number of uncorrelated components, PCA can identify periodic variations that are dominant in the data; each of the components represents a different degree of dominant variability embedded in the data. PCA-based methods in combination with other statistical approaches have been widely used for anomaly detection. Wang and Ong [27], for instance, combined one of the control chart techniques (MEWMA 
control chart) with PCA to detect structural damage using vibrational response. Loh et al. [28] used Singular Spectrum Analysis (SSA), a technique with a similar mathematical basis as PCA, with an autoregressive model to extract the response feature from continuous monitoring of the static deformation of a dam. More recently, Laory et al. [29] applied Moving PCA (MPCA) in combination with four regression analysis methods for damage detection in bridges. The combined methods were compared with stand-alone methods to see which ones provided highest levels of damage detectability as well as earlier detections.

While PCA is often applied to the whole dataset, it can also be applied to a subset, or a window, of the dataset. MPCA performs PCA by sliding this window, so that any change in the principal components over time can be detected. More formally, consider a data matrix, $T \in \mathbb{R}^{N \times M}$, whose $M$ columns are individual time series of length $N$ that have been normalized with respect to each column. Each entry of this matrix can be denoted by $V_{i}(t)$, where $i=1, \ldots, M$ and $t=1, \ldots, N$, as shown in the Eq. (5) below. $V_{i}(t)$ would indicate the measurement of piezometer $i$ at time $t$.

$$
T=\left[\begin{array}{cccc}
V_{1}(1) & V_{2}(1) & \cdots & V_{M}(1) \\
V_{1}(2) & V_{2}(2) & \cdots & V_{M}(2) \\
\vdots & \vdots & \cdots & \vdots \\
V_{1}(N) & V_{2}(N) & \cdots & V_{M}(N)
\end{array}\right]
$$

To apply MPCA on $T$, first a sliding window of size $L$ is applied to the matrix to extract a submatrix, called $R(k)$ at each time value $k$, where $k=1, \ldots,(N-L)$. Then, a singular value decomposition (SVD) is performed on each one of the covariance matrices, $C=\frac{1}{N} R(k)^{T} \times$ $R(k)$. During SVD, the matrix $T$ gets decomposed into matrices $U, S$, and $V$, where $C=U S V^{T}$. The columns of $U$ are the left singular vectors while those of $V$ are the right singular vectors. $S$ is a diagonal matrix with singular values along the diagonal. Since $C$ is symmetric, the right singular vectors correspond to the eigenvectors, $E_{l}$, and the diagonal elements of $S$ correspond to the square roots of the eigenvalues, $e_{l}$, of the matrix (where $l=1, \ldots, L$ ).

The eigenvectors of the covariance matrix represent the directions of maximum variance, or the variance of each independent component, and the corresponding eigenvalue indicates a degree of each component's proportional variance. Thus, the most dominant patterns between reservoir and piezometric readings can be captured by the first few sets of the eigenvectors after ordering the corresponding eigenvalues in a descending order.

\subsection{Kullback-Leibler Divergence}

A nonparametric technique that was tested is based on divergence measurement, which is the distance between two probability densities [30]. One of the most well known divergence estimators is the Kullback-Leibler (KL) divergence [31, 32, 33]. The KL divergence between $p(x)$ and $q(x)$ is expressed in Eq. (6). 
When fitting parameterized models to the samples being tested, various principles can be used to estimate KL divergence [34, 35], e.g., k-nearest neighbors, Maximum Likelihood Method, maximum spacing estimators, etc. In this work, the k-nearest neighbor (kNN) method [34] was adapted to estimate the KL divergence, or distance between the samples from two distributions, $p$ and $q$.

First, the distance between each point in the $d$-dimensional samples, $\left\{X_{i=1, \ldots, n}\right\}$ of $p$ to its $\mathrm{k}^{\text {th }}$ nearest neighbor in $\left\{X_{j}\right\}_{j \neq i}$ are computed. Then, the $\mathrm{k}^{\text {th }}$ nearest neighbor distance of each point from $\left\{X_{i}\right\}$ to the $d$-dimensional samples, $\left\{Y_{l=1, \ldots, m}\right\}$ of $q$ are also computed. Those distances are denoted as $X X_{k}(i)$ and $X Y_{k}(i)$, respectively. The divergence estimator is then expressed as:

$$
\widehat{D}(p, q)=\frac{d}{n} \sum_{i=1}^{n} \log \left(\frac{X Y_{k}(i)}{X X_{k}(i)}\right)+\log \frac{m}{n-1}
$$

In the next section, we will explain how we applied the three statistical techniques in order to improve anomaly detection of piezometer data.

\section{Design of Experiments}

As mentioned in the previous section, the locations of piezometers, including the soil layers in which their tips are installed, as well as different anomaly scenarios influence piezometer responses, and consequently, anomaly detection performance. This section presents two experiments designed to evaluate three different anomaly detection techniques for the selected piezometers of the case study dam (Table 1) and for various anomaly scenarios (Table 2).

For every time series dataset, the first two years of the data were assumed to be free of anomalies (i.e., training data), as our approach requires the definition of a baseline period during which the dam behaves normally. The rest of the data were treated as testing data. In every technique, since piezometers respond to reservoir pool events, both the piezometer readings and the reservoir levels were analyzed together. Thus, given 10 different anomaly scenarios and 10 piezometers, total of 100 2-dimensional datasets, where each dataset is composed of 2-dimensional time series of pool and piezometer, were tested.

The metric used to evaluate the anomaly detection techniques is the $F_{2}$ score, which is a combined measure of precision and recall, but emphasizes recall twice as much as precision (see Eq. (10)). Precision is calculated by the number of correctly labeled anomalies (true positives) divided by the total number of anomalies labeled as belonging to the positive class (i.e., the sum of true positives and false positives) while recall is calculated by the number of correctly 
A control chart was constructed for each technique, where threshold levels were established based on the features extracted from the normal data. Any deviations that went beyond certain sigma levels (standard deviations) of the threshold level were then marked as anomalies. 1 to 3 standard deviations were tested for each technique. It is worth noting that this threshold-based change detection method is sensitive to both gradual and sudden changes.

\subsection{VAR}

In the VAR application, the standard least squares technique was used to determine VAR coefficients and AIC (Akaike's information criterion [36]) to select the order of VAR models. For each dataset, VAR coefficients were estimated from the scaled training data, and residual errors of the testing data (which contain both normal and anomalous data) were calculated. Here, residual errors are the difference between actual and predicted values that are computed from the VAR model developed using the training data. Since reservoir data were given (or do 
not need to be predicted), only the residual errors of the piezometer data were computed. The VAR model still considers both of the previous reservoir and piezometer data to predict new piezometer data.

\subsection{MPCA}

It is necessary to select a window size that is large enough to capture periodicity of the data and not be influenced by measurement noise [29, 37]. Given the daily data, window sizes that range from 365 to 400 were tested. The authors did not test shorter windows in order to capture the entire seasonality ( 1 year) of the data, and did not test larger windows to make sure the numbers of anomalous windows did not exceed those of normal windows.

Once the window size was selected, principal components of the data contained in the window were computed. In our case, we obtained 2 eigenvectors given the 2-dimensional data. As the window was moved towards the end of the time series, any changes in the most dominant pattern was observed only, i.e., first principal component. Since the analyzed data is 2-dimensional (i.e., reservoir level and piezometer reading), the data has two 2-dimensional principal components. We analyzed how the direction (angle) of the first two-dimensional principal component changed over time. Given that eigenvectors are direction cosines for principal components, the cosine angle can be formed by the two elements of each eigenvector. Thus, when observing how the first principal components change over time, the experiment looked for changes in the angles formed by the two elements of the principal component.

The window was moved in 30 day increments instead of one day increments because i) the anomalous problems we are focusing on are slow moving processes, and ii) any anomalies found in one day data are likely caused by noise, and the eigenvectors would not significantly change due to one additional day data. In order to evaluate the detection performance, we observed if the detection technique could detect anomalies as soon as the moving window starts to include at least one anomalous data point. The threshold level of the control chart was established using the mean $+/-1$ to 3 standard deviations of the training data.

\section{$5.3 \mathrm{KL}$}

The kNN method-based KL divergence was applied using the Information Theoretical Estimators (ITE) Toolbox [30]. A k-value of 3 was used. First, the divergence between every window in the training period (i.e., first two years) and the windows in the testing period were computed. Then the median distances computed from the training windows were analyzed to see if there were any significant deviations. For example, let's say there are 20 training windows and 50 testing windows. Then, for each of the 50 testing windows, there would be a total 20 distances computed from the training windows. Then only the median of those 20 distances is saved from each testing window. As the window moves toward the end, the medians (50 of them in total) are examined in order to detect if there are any significant increases. As in the MPCA experiment, 
the window moved by the increments of 30 days, meaning the duration between the two successive moving windows is 30 days, and the detection performance was evaluated by observing if the detection technique could detect anomalous moving window once it contains at least one anomalous data.

In order to establish a threshold level of the control chart, every possible distance among the training windows was computed. The control chart was constructed using the mean of those entire distances being computed, and the standard deviations among the mean distances from each training window to the rest of the training windows. Various threshold levels were obtained from the mean plus three different sigma levels of the standard deviations.

\section{Results}

Table 3 presents the results obtained from VAR based on the best order numbers (please also see Figure 9, which is a heat map generated from this result). The threshold levels, $\sigma$ and the model orders, $\mathrm{M}$, shown in the table correspond to the combinations that bring the best $\mathrm{F}_{2}$ scores. Most piezometers performed badly, while Piezometer 2, 5 and 7 performed comparatively better in extremely severe scenarios (i.e., Scenario 3, 4, 7, 8). The performances on the scenarios that simulate pervious core layer were poor.

Table 4 shows the results of MPCA based on the optimal parameter settings of window sizes, $w$ and threshold levels, $\sigma$ that bring the best $\mathrm{F}_{2}$ scores. Table 5 summarizes the results of KL, also based on the optimal parameter combinations. Figure 10 and 11 are the corresponding heat maps.

In general, the performance of MPCA applied on the graded scenarios was not as good as that applied on the uniform scenarios; the performance of each piezometer improved in Scenario 4 and 8 except Piezometer 10. The results of KL were better compared to MPCA. KL had consistent performance among different piezometers and performed better on the last two scenarios, too (i.e., Scenario 9 and 10).

In real life, it is not possible to determine the optimal parameter settings for anomaly detection especially given that various types of anomalies are not known in advance. Thus, the experiment was conducted to test parameters that may be intuitively selected by those who analyze the data to see how MPCA and KL perform. Given that it is fundamental to inspect annual condition changes, a window size of one year and sigma level of 2 were chosen. However, sigma levels should be appropriately adjusted based on how sensitive your anomaly detection system should be.

Table 6 (Figure 12 vs. 13) clearly shows that KL computed from each piezometer performed better than MPCA on every scenario. 


\section{Discussion}

\subsection{VAR}

As summarized in Table 3, VAR performed very poorly in most piezometers and scenarios (see red colors in Figure 9). It is worth noting that in Scenarios 1, 2, 3, 4 and 9, which are based on 'Duration: Slow', the anomalies were introduced from Jan. 2009 to Mar. 2010 while in Scenarios $5,6,7,8,10$, which are based on 'Duration: Fast', the anomalies were introduced from Jan. 2009 to May 2009.

When VAR did perform well, the scenario was based on severe anomalies that were uniformly introduced (i.e., Scenario 4, 8) along the entire layer within the downstream deposit (not the gradual changes introduced). The upper plot of Figure 14 shows the entire time series (both training and testing data) of PZ 2, and the lower plot of Figure 14 represents the results of AR based on Scenario 4. In the upper plot, the solid vertical line indicates the end of the training period, and the yellow arrow indicates the start of the anomaly introduction. The piezometer readings in red correspond to the simulated piezometer data under Scenario 4 while the predicted readings in blue correspond to the predicted piezometer data using the VAR model that is estimated from the training data. Here, the regression residuals start to exceed the threshold line early enough to capture the anomalies introduced in the first 3 months (Jan. 2009 to Mar. 2009).

However, even based on the same scenario (Scenario 4), when the piezometric response changes were not clearly reflected enough (e.g., PZ 9), the result was not satisfactory as shown in Figure 15 ; the anomalies were detected late, thus leaving many false negatives.

In most cases, the poor performance of VAR was due to significant noise being present in the training data. For example, based on the PZ 1 data from Scenario 3 (Figure 16), slight increases in the regression residuals could be observed during the anomalous period. However, due to a number of fluctuations in the residuals computed from the training data, the threshold level was inadequately established, thus the anomalies could not be accurately detected.

\subsection{MPCA vs. KL}

Under the optimal parameter settings, MPCA performed better than VAR (see Figure 9 vs. 10). There were differences in the performance over different scenarios and piezometers. Relatively, the piezometers installed in the impervious core area seemed to perform poorly when anomalies were introduced in the downstream river deposit. However, their performance improved (even outperformed compared to other piezometers) under the core anomaly scenarios (i.e., Scenario 9 and 10). MPCA seemed incapable of capturing less severe anomalies compared to severe ones as shown in the results based on Scenario 1 vs. Scenario 3 and Scenario 2 vs. Scenario 4. Accordingly, MPCA worked better when the anomalies were uniformly distributed throughout the thin layer, which takes less time to affect further dam areas. 

piezometers performed similarly in each scenario and over different piezometers. In general, the performances of KL on Scenarios 1-4, which are based on 'Duration: Slow', were better than those on Scenarios 5-8, which are based on 'Duration: Fast'. When the results were closely analyzed, the lower $\mathrm{F}_{2}$ scores on Scenario 5-8 were caused by more false positives (not necessarily more false negatives) compared to Scenarios 1-4. Table 6 shows that under the realistic parameter setting, KL also performed better than MPCA.

In Figure 17, the top plot is a scatter plot of PZ 7 readings vs. pool levels. The second plot is the time series of PZ 7. In the time series, the piezometer data that lie in the anomalous period were plotted with plus (+) signs. The third and fourth plots correspond to the results of KL and MPCA, respectively, based on both the normal scenario and Scenario 1 (dashed line). Here, the normal scenario means there are no anomalies introduced throughout the testing period. The results based on this normal condition were presented in order to emphasize the difference in the results of the normal and anomaly scenarios (please note that these normal condition-based results are not the same as the threshold levels used to test the performance of each anomaly detection technique). The KL divergence and the angle computed from MPCA using the data contained in a particular window were indicated with asterisks. The range of this window was also indicated with side arrows in the second plot. The downward arrows in the third and fourth plots indicate the range of the anomalies where certain increases in the KL divergence and MPCA angle are expected. In this figure, it is clearly shown that KL divergence started to increase during the anomalous period while MPCA seemed to fluctuate within the normal range.

Figure 18 shows when both KL and MPCA performed well (Scenario 4). Both techniques show increasing trends within the anomalous period. The asterisks shown in the third and fourth plot correspond to the KL divergence and MPCA angles, respectively when the window contains anomalous data only. As the window slides toward the end, it starts to include the normal data, and both KL and MPCA decrease slowly.

In each KL divergence plot, a sudden increase was observed toward the end, even though such an increase was not expected from the dataset without any anomalies. After closely looking at the data during that particular time period, this increase might be caused by the unusually high pool level (pointed with an arrow) as shown in the left plot of Figure 19. If the pool level suddenly becomes very high, and if the piezometer readings still maintain the same relationship with the pool levels as before, MPCA will be able to correctly label the new piezometer data as normal, because there would be no change in the principal component. However, KL may signal alarms due to the deviated data distribution. Thus, even though this particular case is very unlikely and rare given that sudden pool level changes usually cause seepage-induced lags in piezometer responses, it is important to consider in which conditions the selected techniques would be more appropriate.

While MPCA sometimes showed poor performance when applied to the upstream piezometers (i.e., PZ 1, 2, 3), KL performed well in most cases. As explained before, MPCA looks for changes in the angles formed from the eigenvectors, so in the case of when the anomalous 

such changes are hard to be detected.

Given that KL shows consistently strong results for every piezometer and in every anomaly scenario, KL can be concluded as more sensitive to various anomalies and is capable of handling a more wide range of piezometer locations. As mentioned above, KL is a nonparametric technique that does not make any assumptions on data distribution and linearity. Thus, from this experiment, such assumptions that may be easily made during piezometer data analysis are not always appropriate, and anomaly detection performance can be improved by not making such assumptions about the data.

\section{Conclusion}

In this paper, the applications of three anomaly detection techniques to piezometer data collected from embankment dams were presented in an effort to improve anomaly detection. The piezometer data were simulated using a numerical model called SEEP/W. In this work, two anomalous mechanisms that may possibly be caused by internal erosion were simulated. In the end, 10 various anomaly scenarios were tested.

First, the performance of VAR models was investigated. Since most predictive models perform poorly when there is noise present in data, more robust techniques that extract relevant features from the data were also tested. Most piezometer readings and pool levels are highly correlated, so practicing engineers typically assume that pool and piezometer measurements are linearly related, and are drawn from a normal distribution. However, the analysis showed that depending on various characteristics of piezometers, such assumptions may not always be appropriate in analyzing the data. Thus, both parametric and nonparametric techniques (MPCA and KL, respectively) were investigated in order to compare how they perform in various piezometers and anomaly scenarios. First, various parameter settings (of window sizes and threshold levels) that optimized the performance were tested, where the performance of each method was evaluated using an $\mathrm{F}_{2}$ score. Then, a specific parameter combination was also tested that may be intuitively adopted in real life.

As expected, VAR models were not robust to noise present in the training data, so they performed poorly for most piezometers and in most anomaly scenarios. Both MPCA (parametric), and KL (nonparametric) worked well under the optimal parameters and the specific setting. Under the specified setting, the results of MPCA varied among different piezometers while those of KL were consistent for every piezometer. This implies that KL is sensitive enough to detect even less severe anomalies and applicable to be used in various piezometer locations. Even though it is difficult to state that KL is better than MPCA, this work showed that piezometer data do not always follow linearity and normal distribution, thus the techniques that are based on such assumptions may not always be the best option when analyzing piezometer data. By using nonparametric techniques, the performance of anomaly detection can be improved. 


\section{Acknowledgments}

The work is financially supported by US Army Corps of Engineers.

12

13

14

15

16

17

18

19

20

21

22

23

24

25

26

27

28

29

30

31

32

33

34

35

36

37

38

39

40

41

42

43

44

45

46

47

48

49

50

51

52

53

54

55

56

57

58

59

60

61

62

63

64

65 


\section{References}

[1] ASCE, ASCE | 2013 Report Card for America's Infrastructure | Dams: Conditions \& Capacity, (2013). http://www.infrastructurereportcard.org/a/\#p/dams/conditions-andcapacity (accessed September 4, 2013).

[2] ASCE, ASCE | 2013 Report Card for America's Infrastructure | Grade Sheet: Gpa, (2013). http://www.infrastructurereportcard.org/a/\#p/grade-sheet/gpa (accessed September 4, 2013).

[3] ASDSO, FEMA, Living With Dams: Know Your Risks, (2012).

[4] J. Schurer, E. Wilkinson, J. Norfleet, J.V. Sciver, C. Huntington, C. Lee, et al., Dam Safety Manual, (2002). http://water.state.co.us/DWRIPub/Documents/DS_Manual.pdf.

[5] URBR, Internal Erosion, Dam Safety Risk Analysis Best Practices, (2010). http://www.ferc.gov/industries/hydropower/safety/initiatives/risk-informed-decisionmaking/internal-erosion.pdf.

[6] USSD Committee on Materials for Embankment Dams, The Aging of Embankment Dams, United States Society on Dams (2010).

[7] F. Pelton, Guidelines for Instrumentation and Measurements for Monitoring Dam Performance, (2000). http://cedb.asce.org/cgi/WWWdisplay.cgi?124261 (accessed October 13, 2012).

[8] D. Crum, Rogue Piezometers, 31st Annual USSD Conf. (2011) 1591-1602.

[9] R. Torres, Considerations for Detection of Internal Erosion in Embankment Dams, in: GEO-Velopment, American Society of Civil Engineers (2009) pp. 82-98.

[10] MDEQ, Evaluation of Seepage Conditions, (2007). http://www.deq.state.ms.us/mdeq.nsf/0/DAA3878DB96CD10A8625753800721D52/\$file/E valuation+of+Seepage+Conditions.pdf?OpenElement (accessed October 13, 2013).

[11] R. Fell, C. Wan, J. Cyganiewicz, M. Foster, Time for Development of Internal Erosion and Piping in Embankment Dams, J. Geotech. Geoenvironmental Eng. 129 (2003) 307-314.

[12] Bureau of Reclamation, Best Practices (26. Internal Erosion Risk), (2012).

[13] USBR, Embankment Dams- Chapter 8: Seepage, (2011).

[14] R. Flores-Berrones, N. Patricia, Internal Erosion Due to Water Flow Through Earth Dams and Earth Structures, in: D. Godone (Ed.), Soil Eros. Stud., InTech, (2011).

[15] X. Xu, Sequential anomaly detection based on temporal-difference learning: Principles, models and case studies, Appl. Soft Comput. 10 (2010) 859-867.

[16] V.J. Hodge, J. Austin, A Survey of Outlier Detection Methodologies, Artif. Intell. Rev. 22 (2004) 85-126.

[17] V. Chandola, D. Cheboli, V. Kumar, Detecting Anomalies in a Time Series Database, Computer Science Department, University of Minnesota, (2009). http://www.cs.umn.edu/tech_reports_upload/tr2009/09-004.pdf.

[18] G.P. Zhang, Time series forecasting using a hybrid ARIMA and neural network model, Neurocomputing. 50 (2003) 159-175.

[19] R. Adhikari, R.K. Agrawal, An Introductory Study on Time Series Modeling and Forecasting, LAP LAMBERT Academic Publishing, Saarbrücken, (2013). 
[20] K.K. Nair, A.S. Kiremidjian, K.H. Law, Time series-based damage detection and localization algorithm with application to the ASCE benchmark structure, J. Sound Vib. 291 (2006) 349-368.

[21] P. Omenzetter, J.M.W. Brownjohn, Application of time series analysis for bridge monitoring, Smart Materials and Structures, 15 (2006) 129.

[22] M. Gul, F. Catbas, Damage Assessment with Ambient Vibration Data Using a Novel Time Series Analysis Methodology, J. Struct. Eng. 137 (2011) 1518-1526.

[23] R.H. Shumway, D.S. Stoffer, Time Series Analysis and Its Applications - With R Examples, Third Edition, Springer, (2011).

[24] S. Kay, Modern Spectral Estimation: Theory and Application, 1 edition, Prentice Hall, Englewood Cliffs, N.J., (1999).

[25] S.L. Marple, Digital Spectral Analysis: With Applications, Prentice-Hall, Inc., Upper Saddle River, NJ, USA, (1986).

[26] B. Armour, S.D. Morgera, An exact forward-backward maximum likelihood autoregressive parameter estimation method, IEEE Trans. Signal Process. 39 (1991) 1985-1993.

[27] Z. Wang, K.C.G. Ong, Multivariate Statistical Approach to Structural Damage Detection, J. Eng. Mech. 136 (2010) 12-22.

[28] C.-H. Loh, C.-H. Chen, T.-Y. Hsu, Application of Advanced Statistical Methods for Extracting Long-Term Trends in Static Monitoring Data from an Arch Dam, Struct. Health Monit. (2011).

[29] I. Laory, T. Trinh, D. Posenato, I. Smith, Combined Model-Free Data-Interpretation Methodologies for Damage Detection During Continuous Monitoring of Structures, J. Comput. Civ. Eng. (2013).

[30] Z. Szabo, Information Theoretical Estimators Toolbox (2014). http://arxiv.org/abs/1405.2106 (accessed August 8, 2014).

[31] S. Kullback, R.A. Leibler, On Information and Sufficiency, Ann. Math. Stat. 22 (1951) 7986.

[32] C.M. Bishop, Pattern Recognition and Machine Learning, Springer, New York, (2007).

[33] D.D. Polani, Kullback-Leibler Divergence, in: W. Dubitzky, O. Wolkenhauer, K.-H. Cho, H. Yokota (Eds.), Encycl. Syst. Biol., Springer New York, (2013) pp. 1087-1088.

[34] Q. Wang, S.R. Kulkarni, S. Verdu, Divergence Estimation for Multidimensional Densities Via -Nearest-Neighbor Distances, IEEE Trans. Inf. Theory. 55 (2009) 2392-2405.

[35] M. Basseville, Review: Divergence Measures for Statistical Data processing-An Annotated Bibliography, Signal Process. 93 (2013) 621-633.

[36] K.P. Burnham, D.R. Anderson, Model Selection and Multimodel Inference: A Practical Information-Theoretic Approach, Springer Science \& Business Media, (2002).

[37] D. Posenato, F. Lanata, D. Inaudi, I.F.C. Smith, Model-free data interpretation for continuous monitoring of complex structures, Adv. Eng. Inform. 22 (2008) 135-144. 


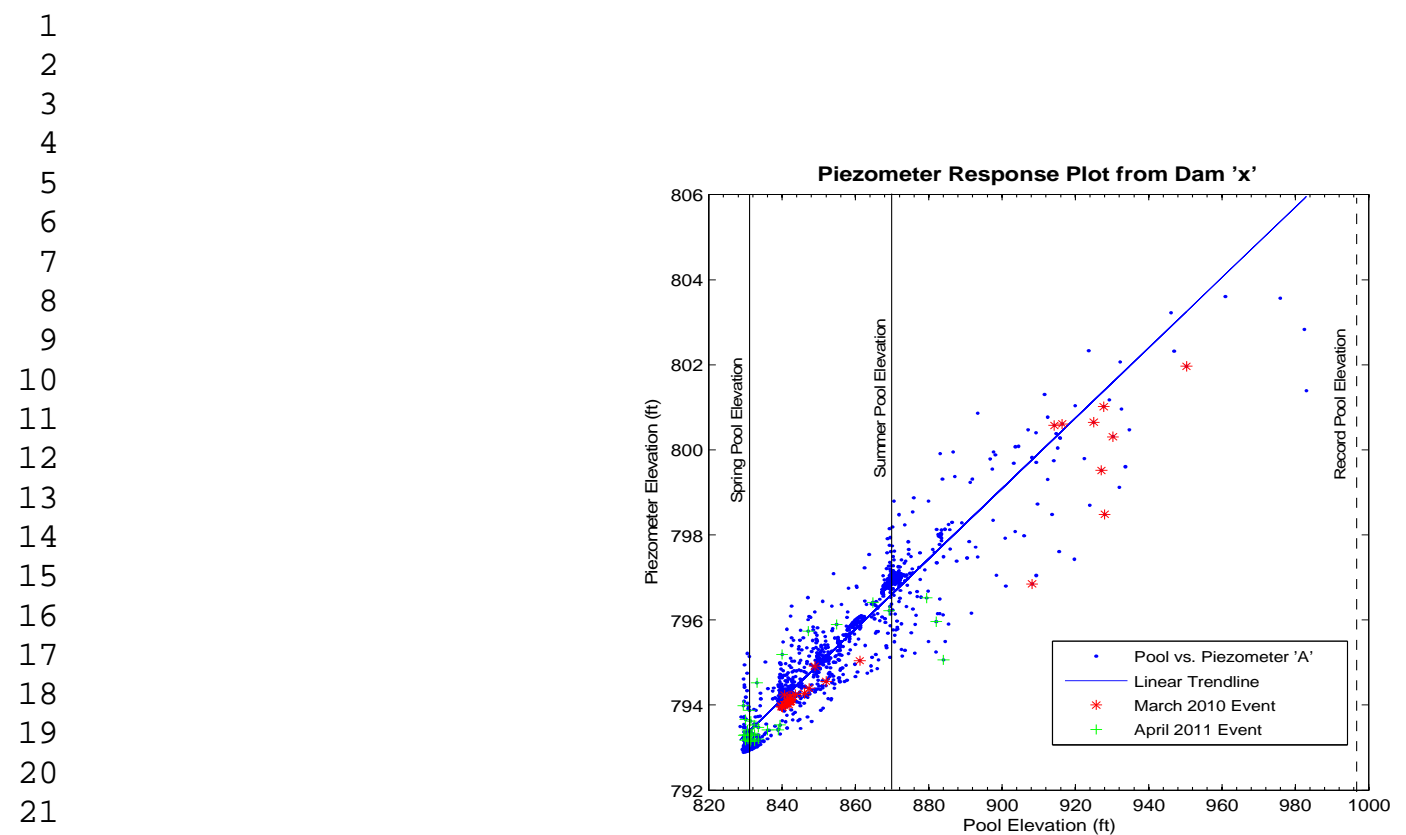

Fig. 1. Traditional correlation plot (pool vs. piezometer) 


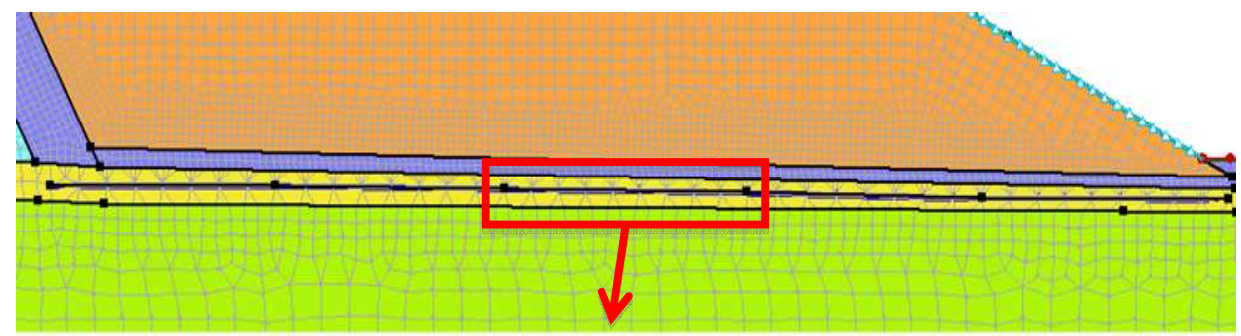

\section{1}

2

3

4

5

Fig. 4. Zoom-in version of Figure 3 around the red line that corresponds to the thin layer with 5 segments, which was created to simulate the foundation erosion problem 
Duration

(months)

$1 \sim 3$

\begin{tabular}{|c|c|c|c|c|}
\hline original & original & original & original & $2 *$ original \\
\hline
\end{tabular}

$4 \sim 6$

\begin{tabular}{|l|l|l|l|l|}
\hline original & original & original & $2 *$ original & $4 *$ original \\
\hline
\end{tabular}

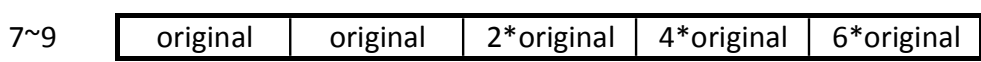

\begin{tabular}{|c|c|c|c|c|c|}
\hline 9 12 & original & $2 *$ original & $4 *$ original & $6 *$ original & $8^{*}$ original \\
\hline
\end{tabular}

\begin{tabular}{|l|l|l|l|l|}
\hline $2 *$ original & $4 *$ original & $6 *$ original & $8 *$ original & $10^{*}$ original \\
\hline
\end{tabular}

\section{Fig. 5. Simulation illustration based on Scenario 1 ('Severity: Low, Duration: Slow, and} Uniformity: Graded')
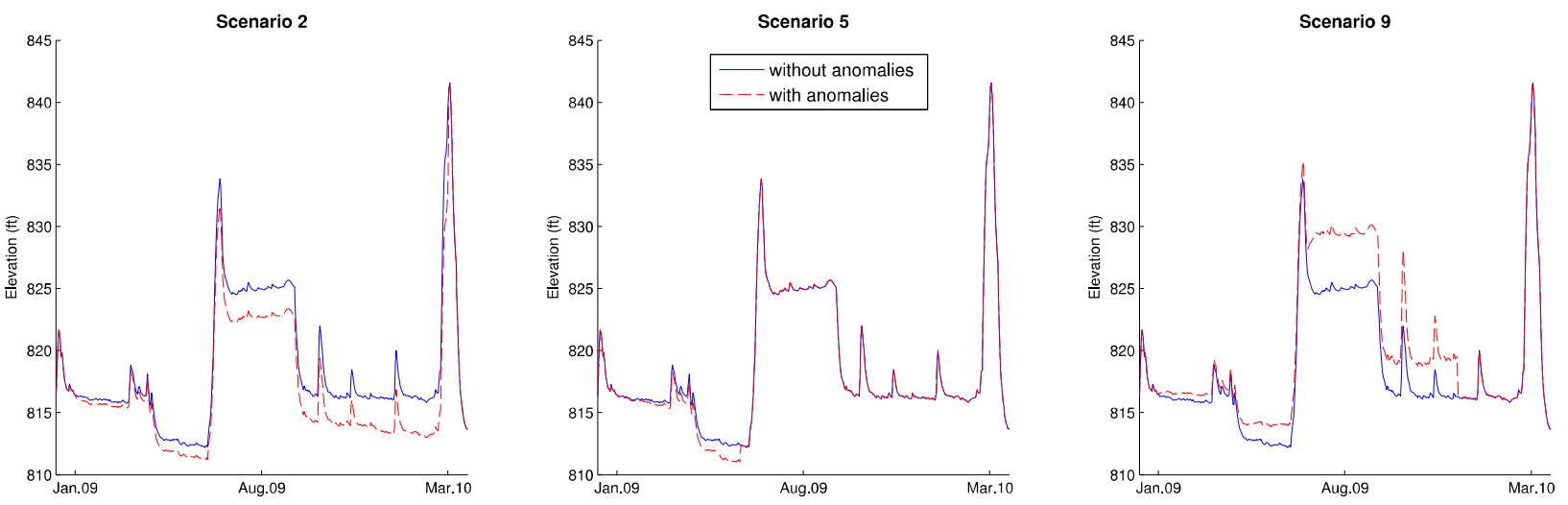

Fig. 6. Parts of the simulated PZ 4 time series based on various scenarios 

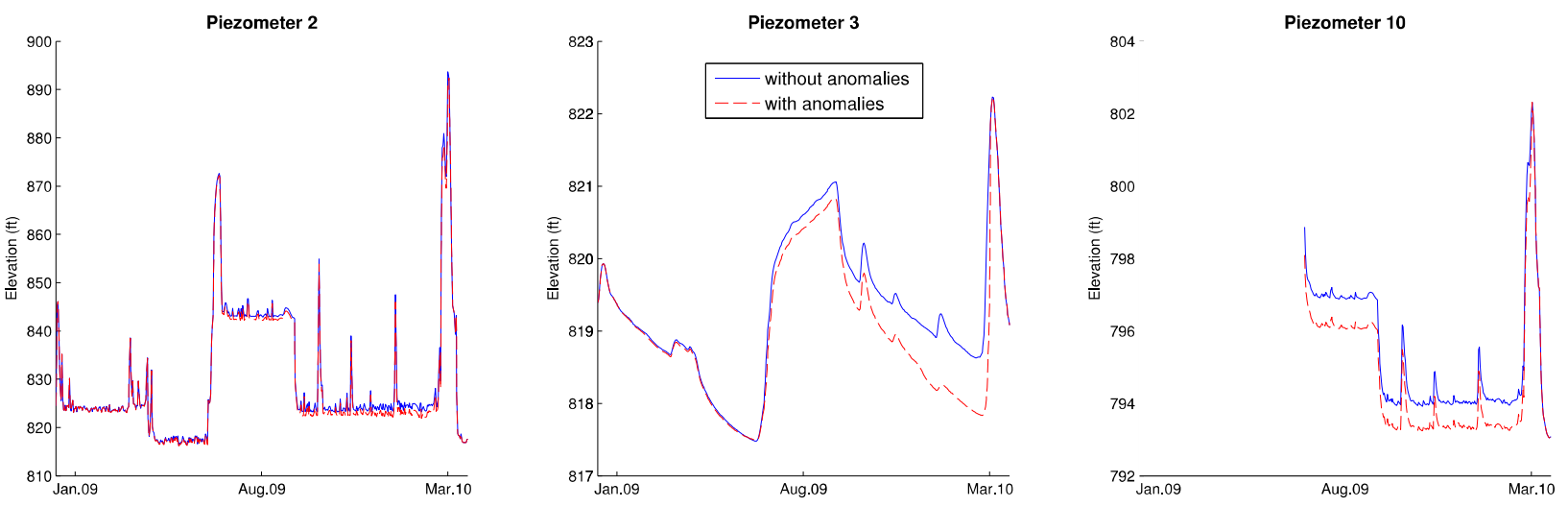

Fig. 7. Parts of the simulated anomalous time series based on Scenario 1

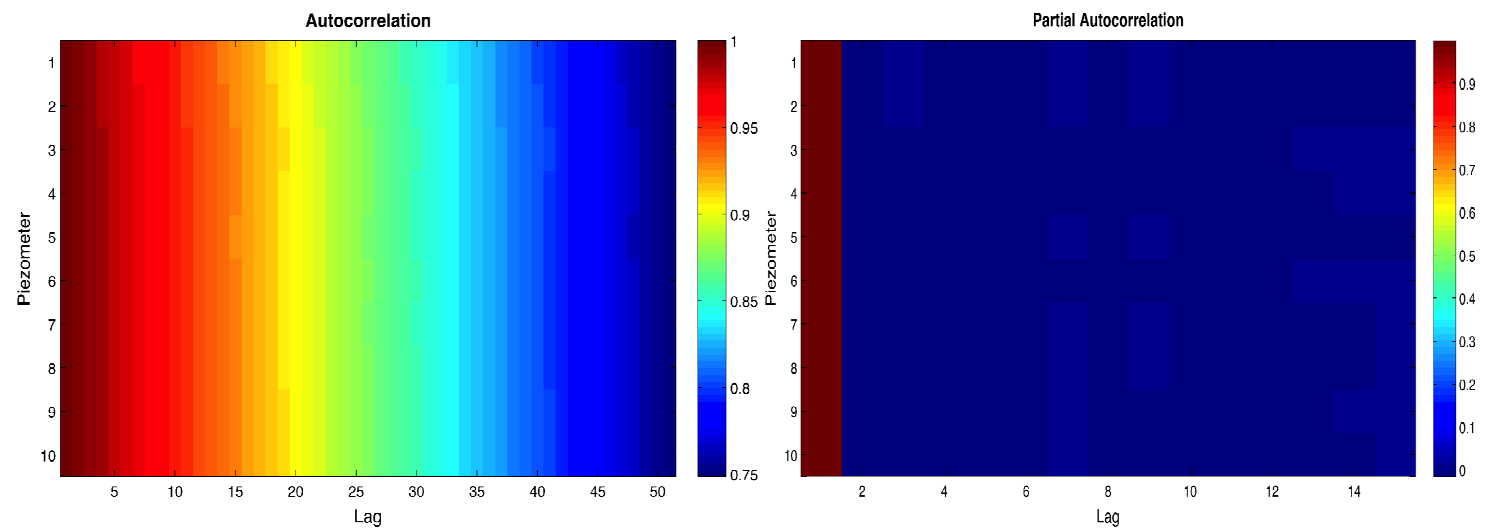

Fig. 8. ACF and PACF of 10 piezometers 


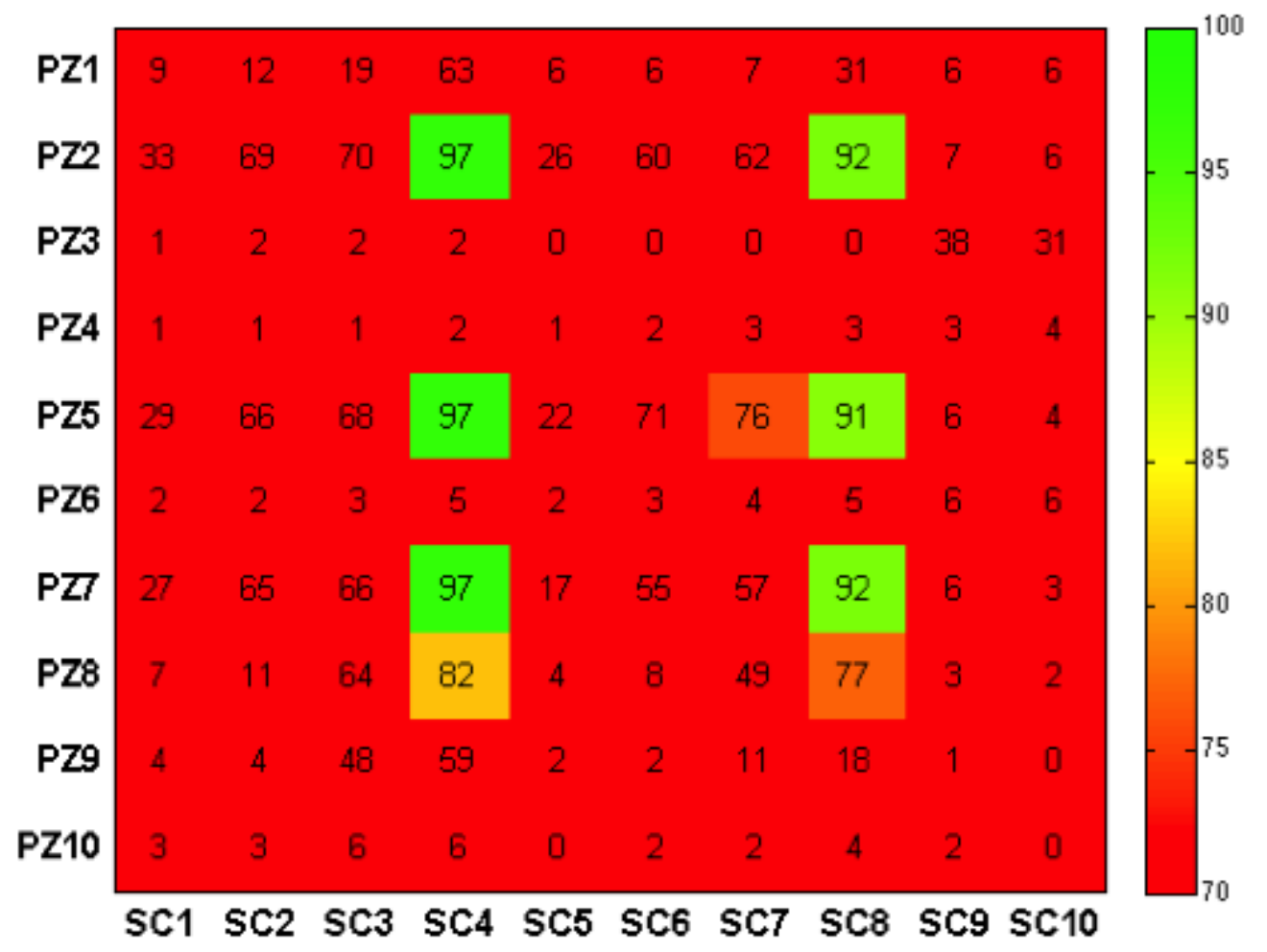

Fig. 9. Heat map for $F_{2}$ values generated from the VAR results shown in Table 3

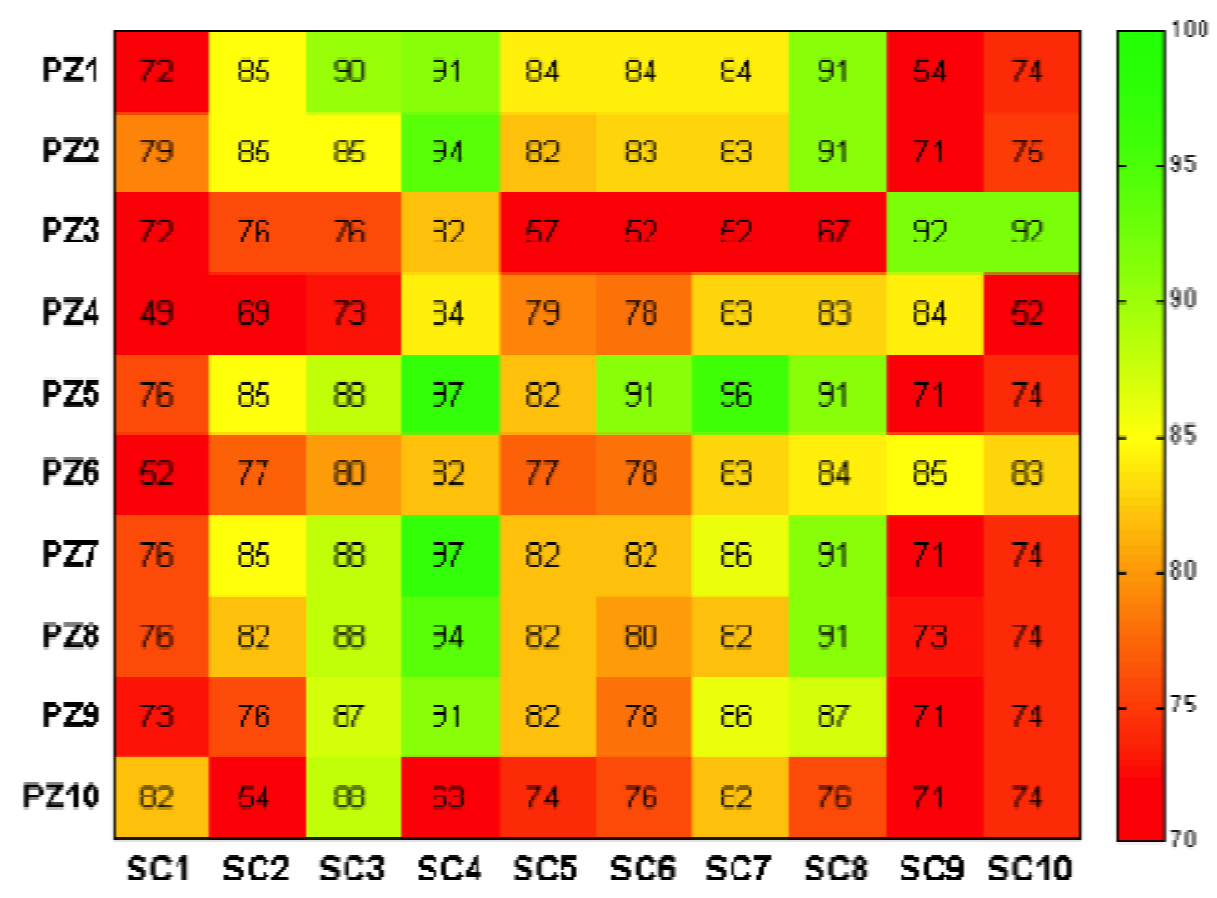

Fig. 10. Heat map for $F_{2}$ values generated from the MPCA results shown in Table 4 
Fig. 11. Heat map for $F_{2}$ values generated from the $K L$ results shown in Table 5

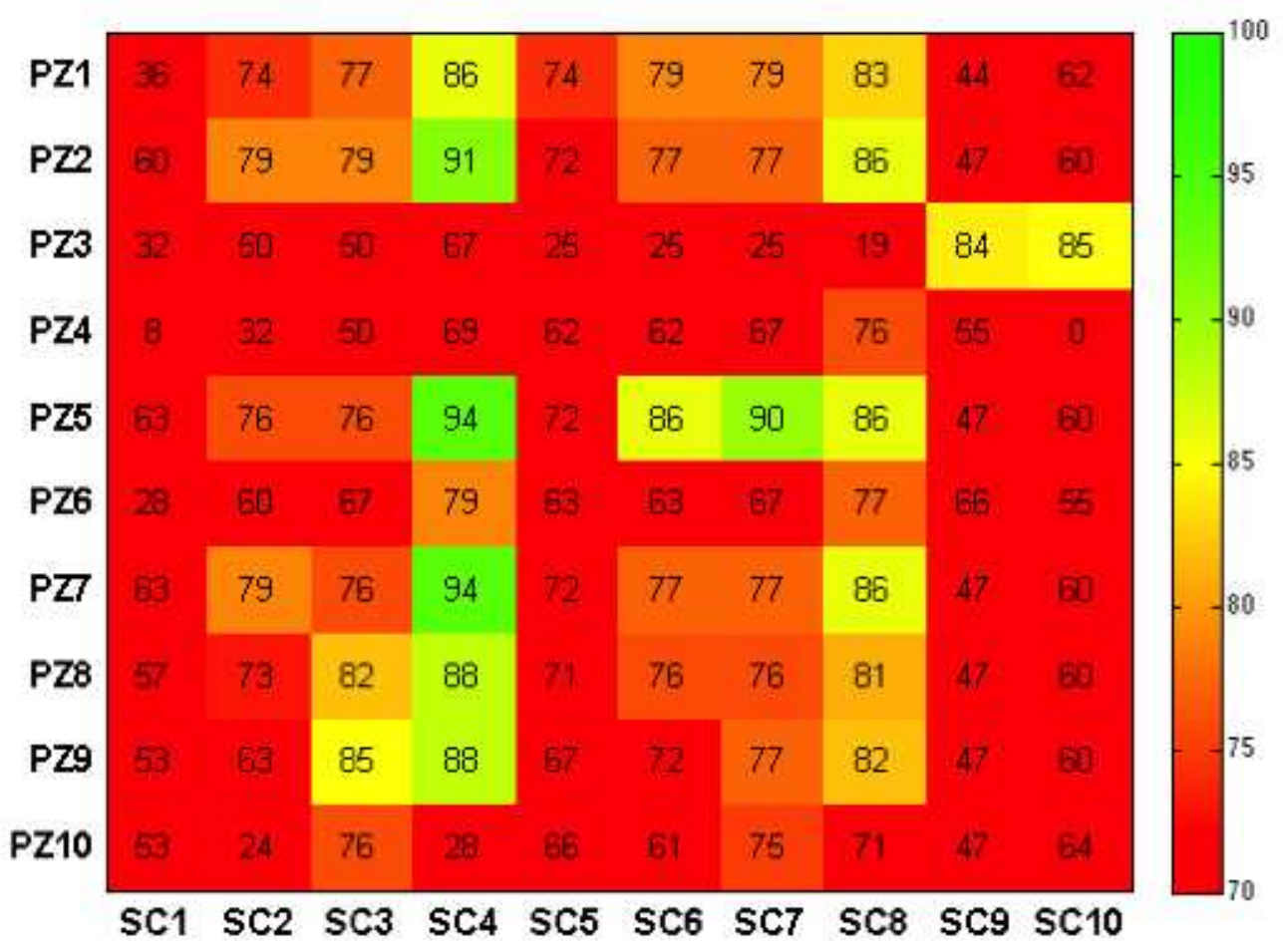

Fig. 12. Heat map for F2 values generated from the MPCA results shown in Table 6 


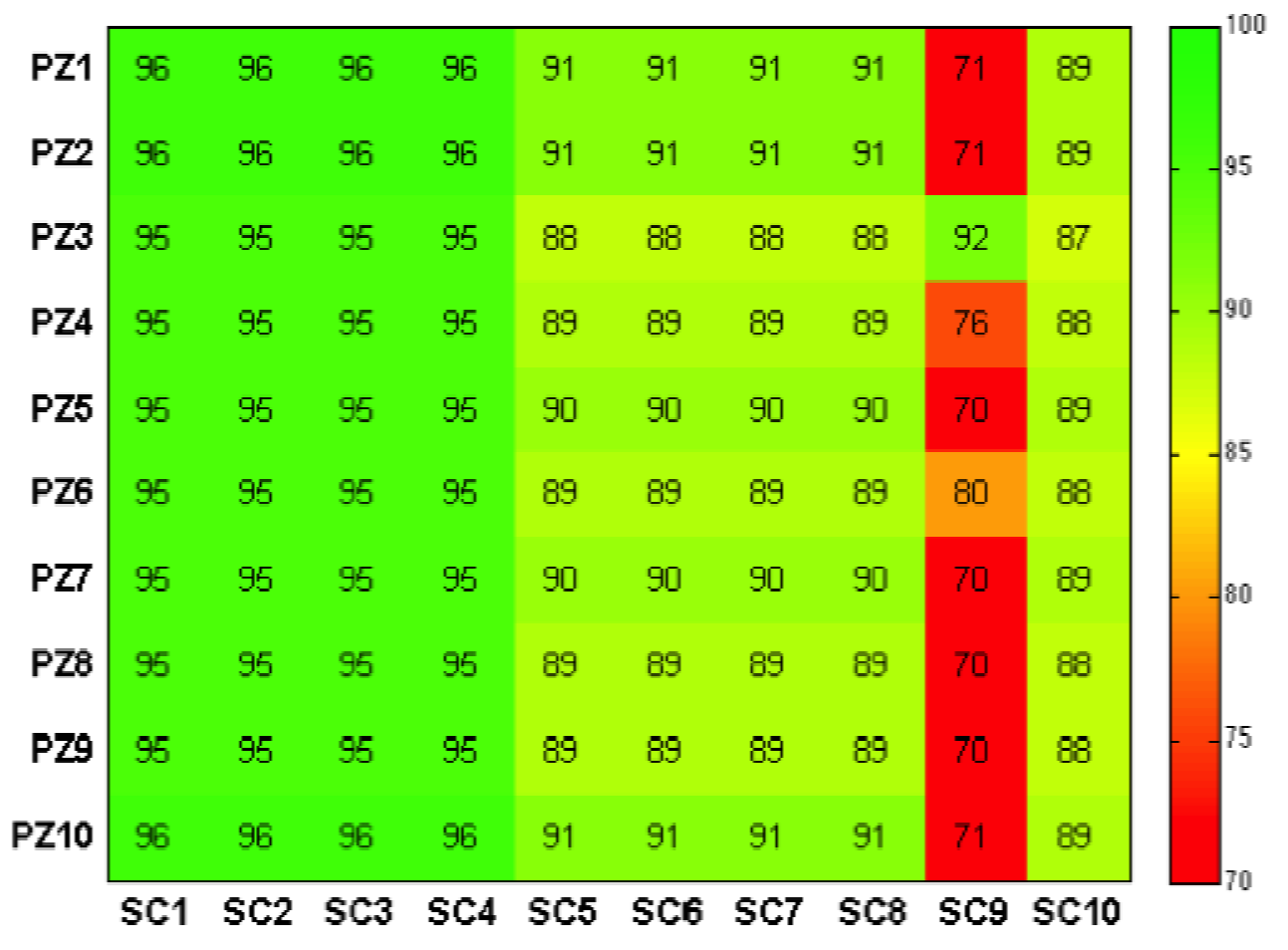

Fig. 13. Heat map for $F 2$ values generated from the KL results shown in Table 6 

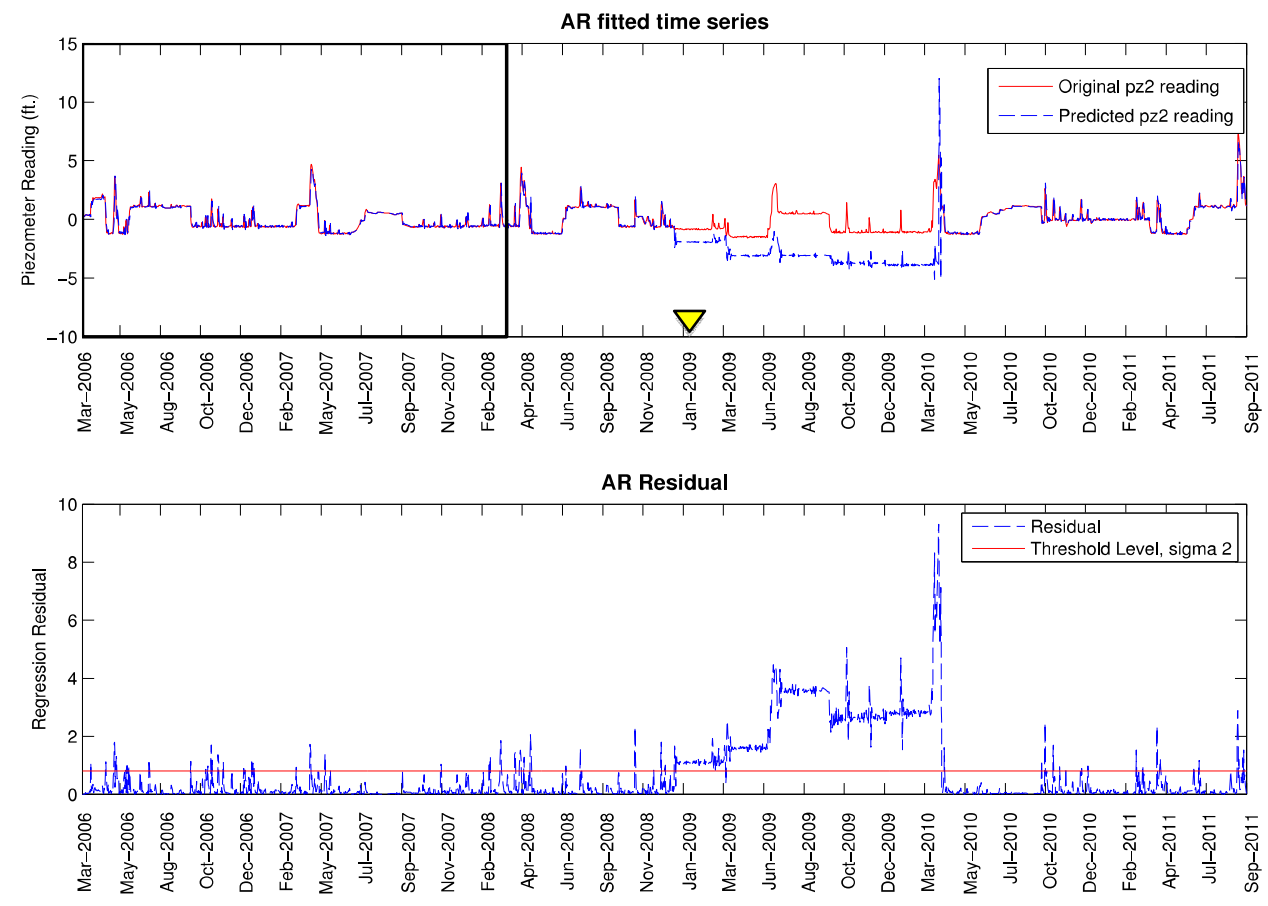

Fig. 14. Result of AR based on Scenario 4 and PZ 2 

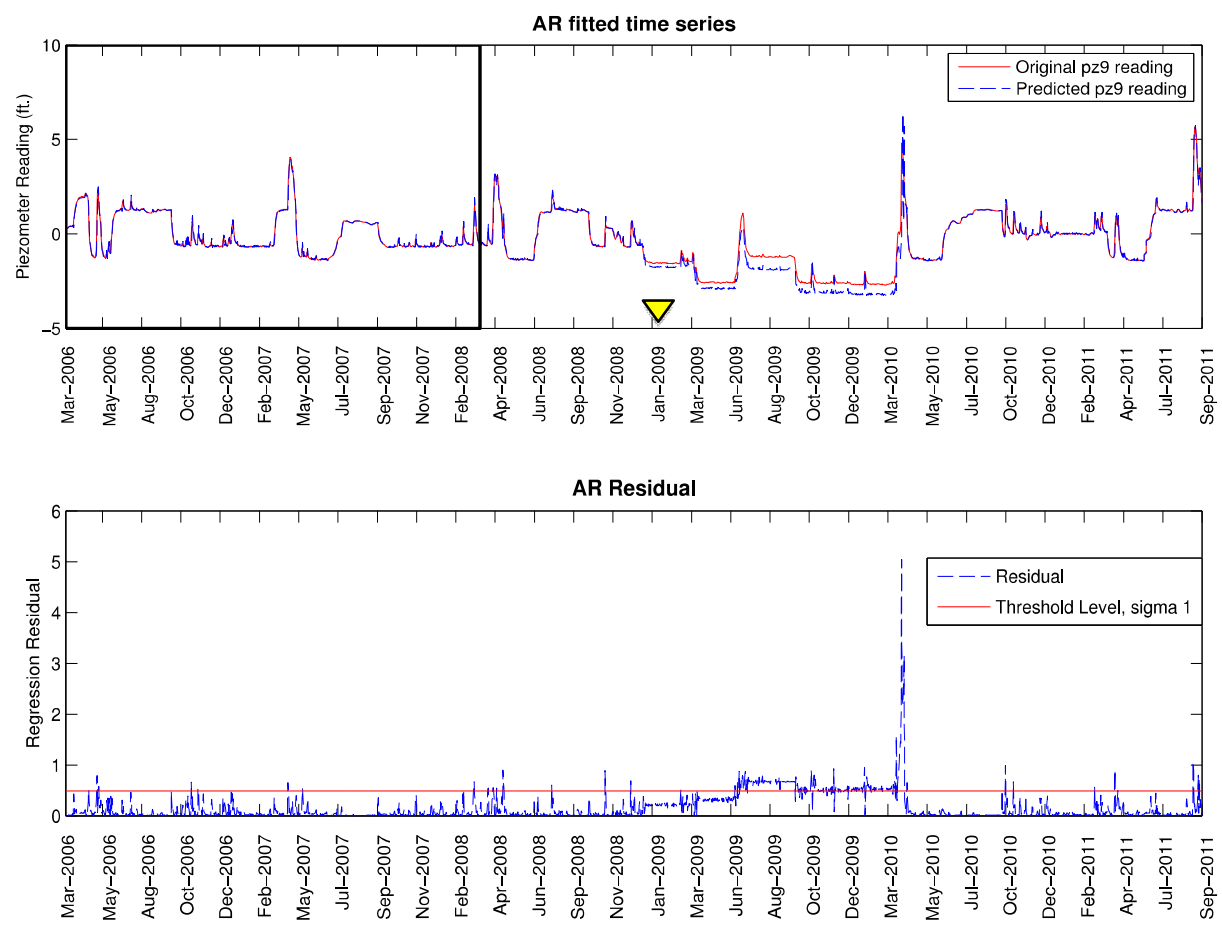

Fig. 15. Result of AR based on Scenario 4 and PZ 9 

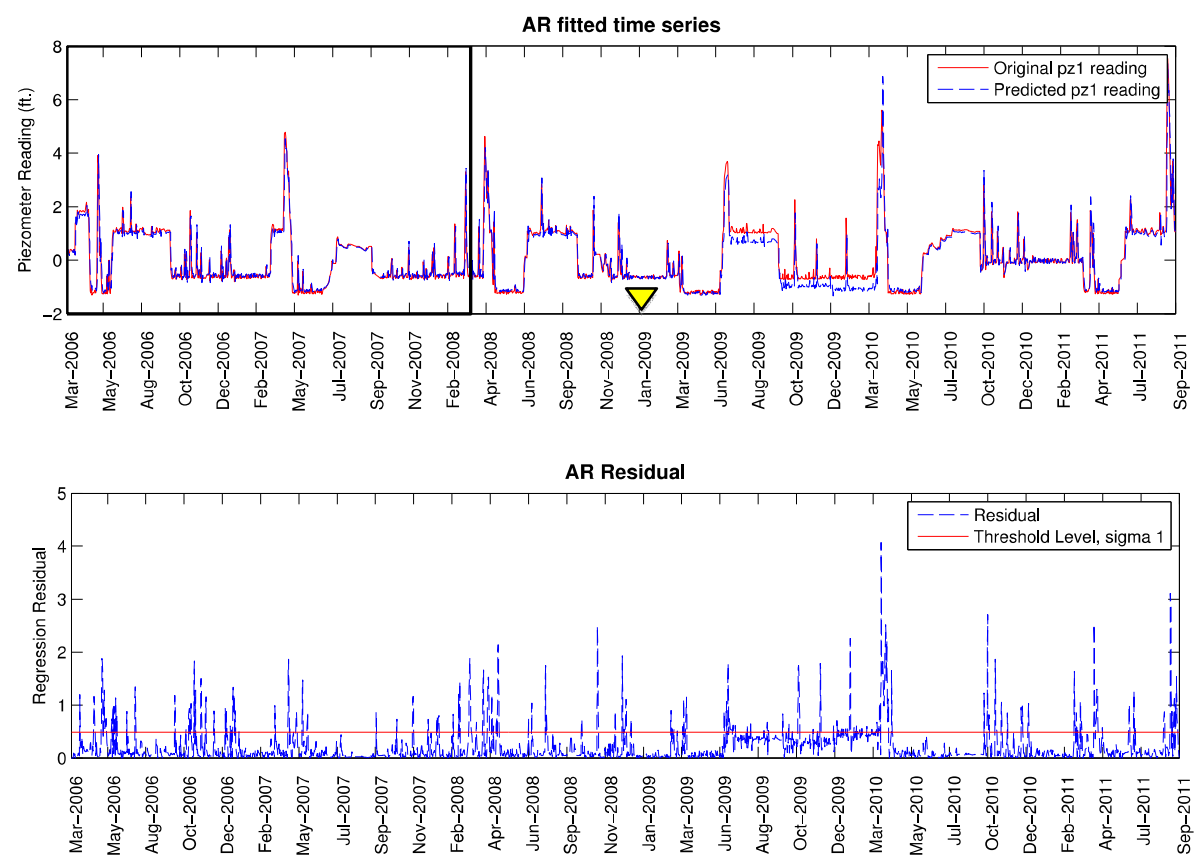

Fig. 16. Result of AR based on Scenario 3 and PZ 1 

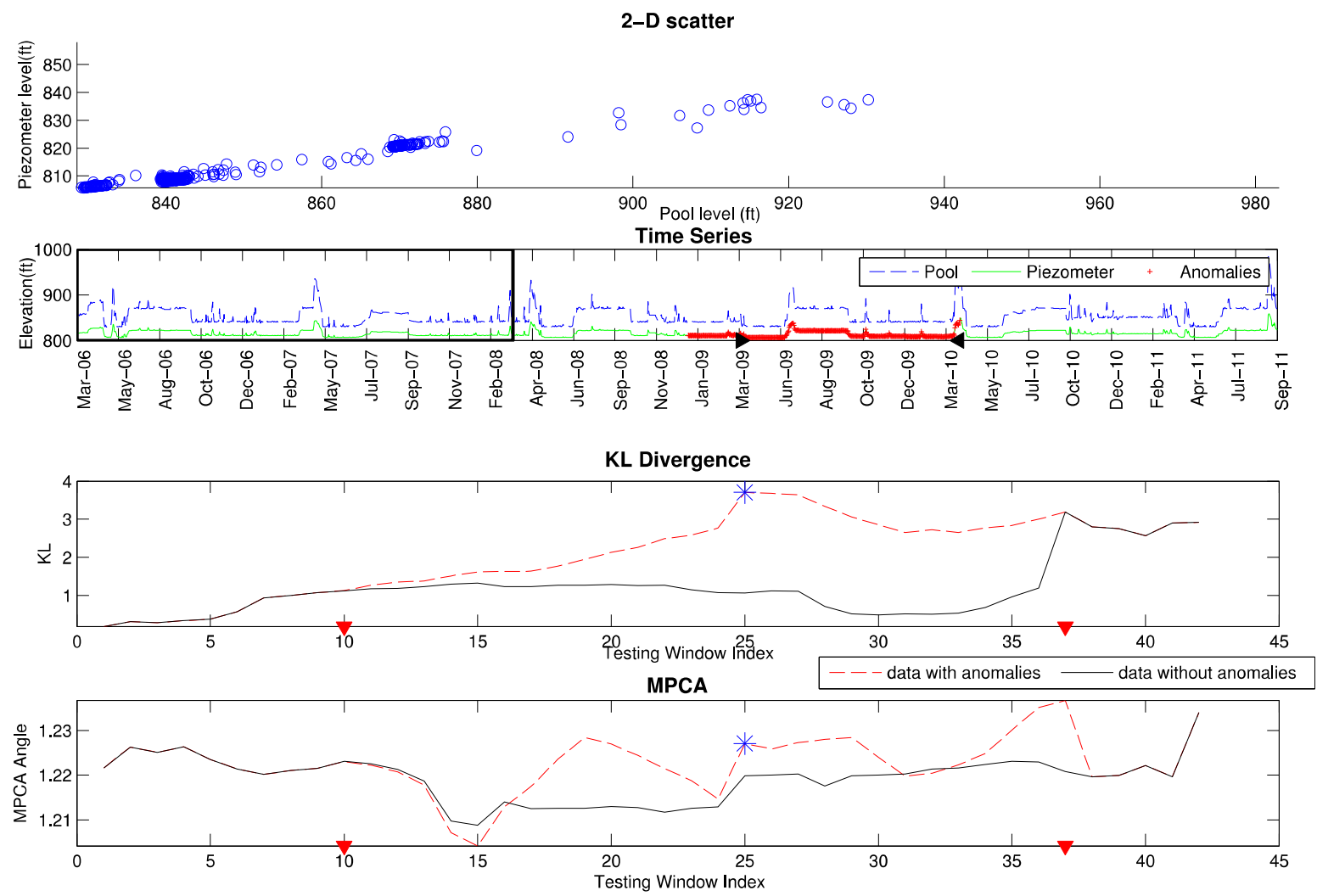

Fig. 17. Results of KL and MPCA (window size 365, Scenario 1, PZ 7) 

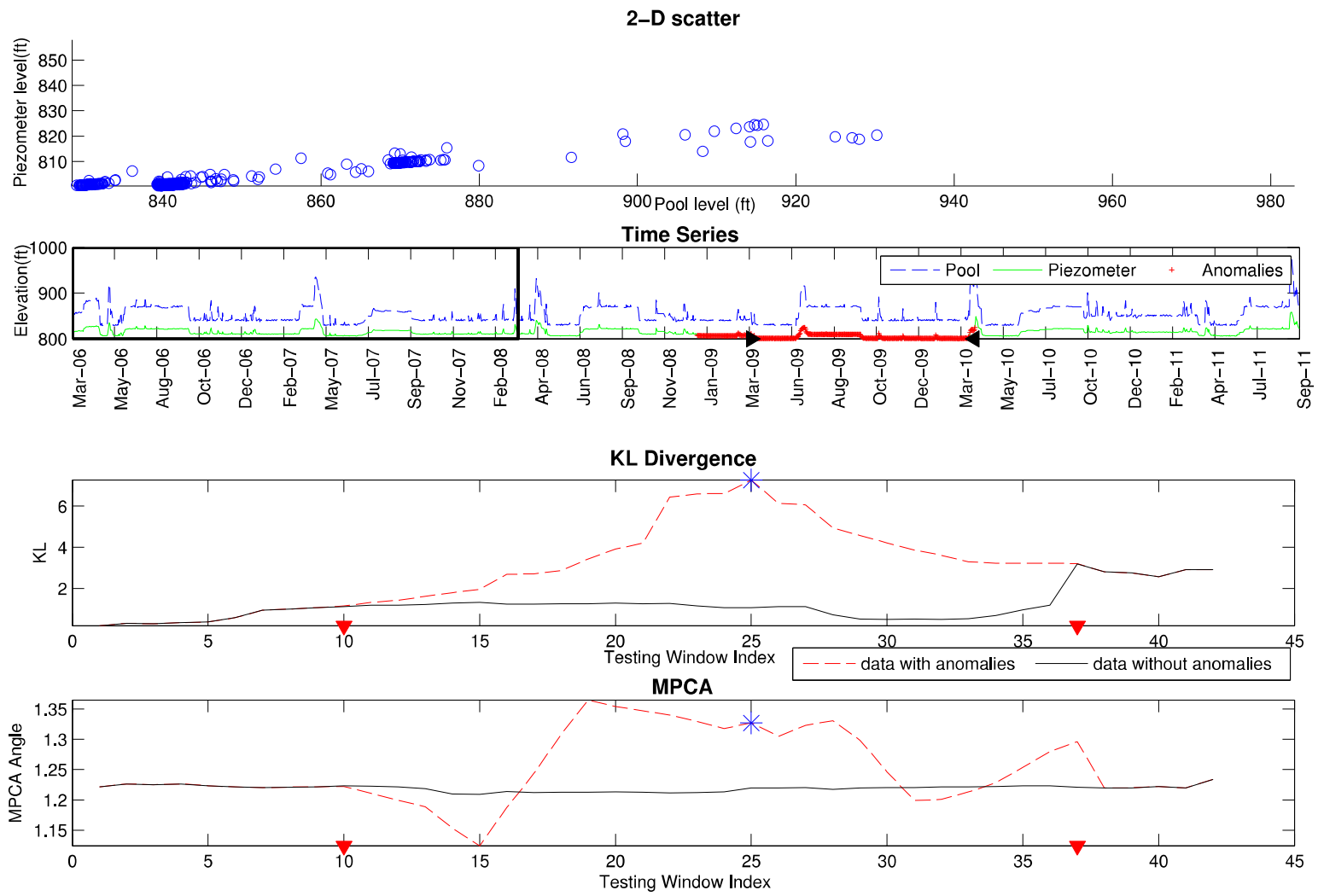

Fig. 18. Results of KL and MPCA (window size 365, Scenario 4, PZ 7)
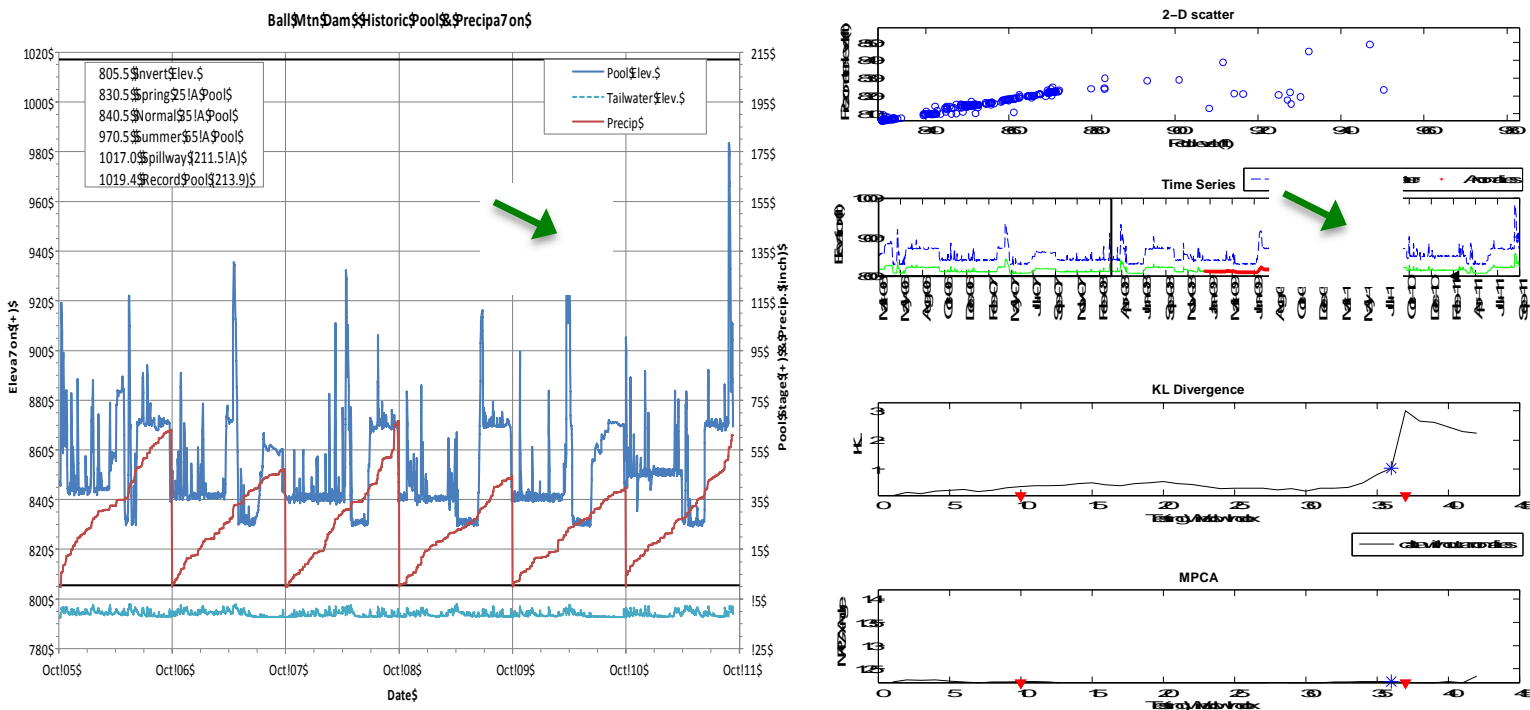

Fig. 19. Pool information (left); Results of KL and MPCA (right)

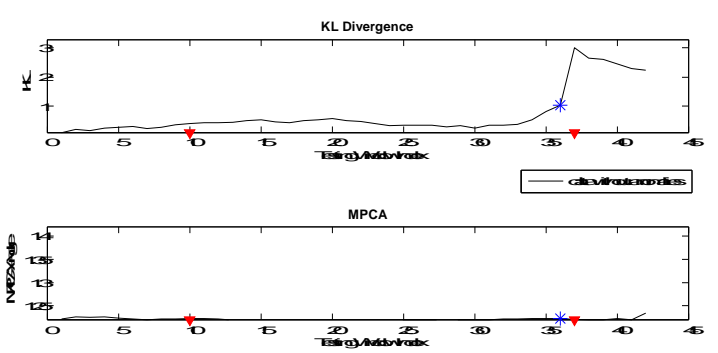


Table 1. Properties of the dam at piezometer locations

\begin{tabular}{ccccc}
\hline Piezometer & $\begin{array}{c}\text { X-coordinate } \\
\text { (ft.) }\end{array}$ & $\begin{array}{c}\text { Y-coordinate } \\
(\mathbf{f t} .)\end{array}$ & $\begin{array}{c}\text { Material/ } \\
\text { Region }\end{array}$ & $\begin{array}{c}\text { Hydraulic } \\
\text { Conductivity } \\
\text { (ft./s) }\end{array}$ \\
\hline PZ 1 & -434.908 & 788.4537 & River Deposit & 0.007 \\
\hline PZ 2 & -198.799 & 784.7021 & River Deposit & 0.007 \\
\hline PZ 3 & -8.8836 & 818.8395 & Impervious Core & $8 \mathrm{e}-008$ \\
\hline PZ 4 & -8.8836 & 800.683 & Impervious Core & $8 \mathrm{e}-008$ \\
\hline PZ 5 & -8.9629 & 785.4531 & River Deposit & 0.007 \\
\hline PZ 6 & 71.1705 & 801.7607 & Impervious Core & $8 \mathrm{e}-008$ \\
\hline PZ 7 & 63.24691 & 788.2472 & River Deposit & 0.007 \\
\hline PZ 8 & 200.6962 & 787.4987 & DS Gravel & 0.0002 \\
\hline PZ 9 & 300.8705 & 795.7294 & Rockfill & 0.002 \\
\hline PZ 10 & 415.5292 & 775.7503 & River Deposit & 0.007 \\
\hline
\end{tabular}

Table 2. Anomaly scenarios

\begin{tabular}{ccccc}
\hline Scenario & Location & Severity & Duration & Uniformity \\
\hline 1 & River Deposit & Low & Slow & Graded \\
2 & River Deposit & Low & Slow & Uniform \\
3 & River Deposit & High & Slow & Graded \\
4 & River Deposit & High & Slow & Uniform \\
5 & River Deposit & Low & Fast & Graded \\
6 & River Deposit & Low & Fast & Uniform \\
7 & River Deposit & High & Fast & Graded \\
8 & River Deposit & High & Fast & Uniform \\
9 & Core & e-High & Slow & Uniform \\
10 & Core & e-High & Fast & Uniform \\
\hline
\end{tabular}


Table 3. Results of VAR based on optimal parameters 
Table 4. Results of MPCA based on optimal parameters 
Table 5. Results of KL based on optimal parameters

\begin{tabular}{|c|c|c|c|c|c|c|c|c|c|c|c|c|c|c|c|c|}
\hline & \multicolumn{3}{|c|}{ Scenario 1 } & \multicolumn{3}{|c|}{ Scenario 2 } & \multicolumn{3}{|c|}{ Scenario 3 } & \multicolumn{3}{|c|}{ Scenario 4 } & \multicolumn{3}{|c|}{ Scenario 5 } \\
\hline PZ & $w$ & $\sigma$ & $\mathrm{F}_{2}$ & $w$ & $\sigma$ & $\mathrm{F}_{2}$ & $w$ & $\sigma$ & $\mathrm{F}_{2}$ & $w$ & $\sigma$ & $\mathrm{F}_{2}$ & $w$ & $\sigma$ & $\mathrm{F}_{2}$ \\
\hline $\mathbf{1}$ & 395 & 2 & $97 \%$ & 395 & 2 & $97 \%$ & 395 & 2 & $97 \%$ & 395 & 2 & $97 \%$ & 380 & 2 & $92 \%$ \\
\hline $\mathbf{2}$ & 400 & 2 & $97 \%$ & 400 & 2 & $97 \%$ & 400 & 2 & $97 \%$ & 400 & 2 & $97 \%$ & 370 & 2 & $92 \%$ \\
\hline $\mathbf{3}$ & 395 & 2 & $96 \%$ & 385 & 3 & $97 \%$ & 395 & 2 & $96 \%$ & 395 & 3 & $97 \%$ & 390 & 2 & $89 \%$ \\
\hline $\mathbf{4}$ & 395 & 1 & $96 \%$ & 395 & 1 & $96 \%$ & 395 & 1 & $96 \%$ & 395 & 1 & $96 \%$ & 395 & 1 & $90 \%$ \\
\hline $\mathbf{5}$ & 370 & 2 & $97 \%$ & 385 & 3 & $97 \%$ & 385 & 3 & $97 \%$ & 385 & 3 & $97 \%$ & 370 & 2 & $92 \%$ \\
\hline $\mathbf{6}$ & 395 & 1 & $96 \%$ & 395 & 1 & $96 \%$ & 395 & 1 & $96 \%$ & 385 & 3 & $97 \%$ & 370 & 2 & $90 \%$ \\
\hline $\mathbf{7}$ & 370 & 2 & $97 \%$ & 385 & 3 & $97 \%$ & 385 & 3 & $97 \%$ & 385 & 3 & $97 \%$ & 370 & 2 & $92 \%$ \\
\hline $\mathbf{8}$ & 370 & 2 & $97 \%$ & 390 & 3 & $97 \%$ & 390 & 3 & $97 \%$ & 385 & 3 & $97 \%$ & 370 & 2 & $92 \%$ \\
\hline $\mathbf{9}$ & 370 & 2 & $97 \%$ & 370 & 2 & $97 \%$ & 370 & 2 & $97 \%$ & 390 & 3 & $97 \%$ & 370 & 2 & $92 \%$ \\
\hline $\mathbf{1 0}$ & 395 & 2 & $97 \%$ & 395 & 2 & $97 \%$ & 390 & 3 & $97 \%$ & 395 & 2 & $97 \%$ & 370 & 2 & $92 \%$ \\
\hline & Scenario 6 & Scenario 7 & Scenario 8 & Scenario 9 & Scenario 10 \\
\hline PZ & $w$ & $\sigma$ & $\mathrm{F}_{2}$ & $w$ & $\sigma$ & $\mathrm{F}_{2}$ & $w$ & $\sigma$ & $\mathrm{F}_{2}$ & $w$ & $\sigma$ & $\mathrm{F}_{2}$ & $w$ & $\sigma$ & $\mathrm{F}_{2}$ \\
\hline $\mathbf{1}$ & 380 & 2 & $92 \%$ & 380 & 2 & $92 \%$ & 380 & 2 & $92 \%$ & 395 & 1 & $75 \%$ & 395 & 2 & $93 \%$ \\
\hline $\mathbf{2}$ & 370 & 2 & $92 \%$ & 370 & 2 & $92 \%$ & 370 & 2 & $92 \%$ & 400 & 1 & $78 \%$ & 400 & 2 & $93 \%$ \\
\hline $\mathbf{3}$ & 390 & 2 & $89 \%$ & 390 & 2 & $89 \%$ & 390 & 2 & $89 \%$ & 380 & 3 & $94 \%$ & 395 & 3 & $93 \%$ \\
\hline $\mathbf{4}$ & 395 & 1 & $90 \%$ & 395 & 1 & $90 \%$ & 395 & 1 & $90 \%$ & 395 & 1 & $94 \%$ & 395 & 2 & $91 \%$ \\
\hline $\mathbf{5}$ & 370 & 2 & $92 \%$ & 370 & 2 & $92 \%$ & 370 & 2 & $92 \%$ & 395 & 1 & $81 \%$ & 395 & 2 & $91 \%$ \\
\hline $\mathbf{6}$ & 370 & 2 & $90 \%$ & 370 & 2 & $90 \%$ & 370 & 2 & $90 \%$ & 395 & 1 & $94 \%$ & 395 & 2 & $91 \%$ \\
\hline $\mathbf{7}$ & 370 & 2 & $92 \%$ & 370 & 2 & $92 \%$ & 370 & 2 & $92 \%$ & 395 & 1 & $81 \%$ & 395 & 2 & $91 \%$ \\
\hline $\mathbf{8}$ & 370 & 2 & $92 \%$ & 370 & 2 & $92 \%$ & 370 & 2 & $92 \%$ & 395 & 1 & $81 \%$ & 395 & 2 & $91 \%$ \\
\hline $\mathbf{9}$ & 370 & 2 & $92 \%$ & 370 & 2 & $92 \%$ & 370 & 2 & $92 \%$ & 400 & 1 & $81 \%$ & 395 & 2 & $91 \%$ \\
\hline $\mathbf{1 0}$ & 370 & 2 & $92 \%$ & 370 & 2 & $92 \%$ & 370 & 2 & $92 \%$ & 400 & 1 & $81 \%$ & 395 & 2 & $92 \%$ \\
\hline
\end{tabular}


Table 6. Results of MPCA and KL using a window size of 365 and a sigma level of 2

\begin{tabular}{|c|c|c|c|c|c|c|c|c|c|c|}
\hline & \multicolumn{2}{|c|}{ Scenario 1 } & \multicolumn{2}{|c|}{ Scenario 2 } & \multicolumn{2}{c|}{ Scenario 3 } & \multicolumn{2}{c|}{ Scenario 4 } & \multicolumn{2}{c|}{ Scenario 5 } \\
\hline PZ & MPCA & KL & MPCA & KL & MPCA & KL & MPCA & KL & MPCA & KL \\
\hline $\mathbf{1}$ & $36 \%$ & $96 \%$ & $74 \%$ & $96 \%$ & $77 \%$ & $96 \%$ & $86 \%$ & $96 \%$ & $74 \%$ & $91 \%$ \\
\hline $\mathbf{2}$ & $60 \%$ & $96 \%$ & $79 \%$ & $96 \%$ & $79 \%$ & $96 \%$ & $91 \%$ & $96 \%$ & $72 \%$ & $91 \%$ \\
\hline $\mathbf{3}$ & $32 \%$ & $95 \%$ & $50 \%$ & $95 \%$ & $50 \%$ & $95 \%$ & $67 \%$ & $95 \%$ & $25 \%$ & $88 \%$ \\
\hline $\mathbf{4}$ & $8 \%$ & $95 \%$ & $32 \%$ & $95 \%$ & $50 \%$ & $95 \%$ & $69 \%$ & $95 \%$ & $62 \%$ & $89 \%$ \\
\hline $\mathbf{5}$ & $63 \%$ & $95 \%$ & $76 \%$ & $95 \%$ & $76 \%$ & $95 \%$ & $94 \%$ & $95 \%$ & $72 \%$ & $90 \%$ \\
\hline $\mathbf{6}$ & $28 \%$ & $95 \%$ & $60 \%$ & $95 \%$ & $67 \%$ & $95 \%$ & $79 \%$ & $95 \%$ & $63 \%$ & $89 \%$ \\
\hline $\mathbf{7}$ & $63 \%$ & $95 \%$ & $79 \%$ & $95 \%$ & $76 \%$ & $95 \%$ & $94 \%$ & $95 \%$ & $72 \%$ & $90 \%$ \\
\hline $\mathbf{8}$ & $57 \%$ & $95 \%$ & $73 \%$ & $95 \%$ & $82 \%$ & $95 \%$ & $88 \%$ & $95 \%$ & $71 \%$ & $89 \%$ \\
\hline $\mathbf{9}$ & $53 \%$ & $95 \%$ & $63 \%$ & $95 \%$ & $85 \%$ & $95 \%$ & $88 \%$ & $95 \%$ & $67 \%$ & $89 \%$ \\
\hline $\mathbf{1 0}$ & $53 \%$ & $96 \%$ & $24 \%$ & $96 \%$ & $76 \%$ & $96 \%$ & $28 \%$ & $96 \%$ & $66 \%$ & $91 \%$ \\
\hline & Scenario 6 & Scenario 7 & Scenario 8 & Scenario 9 & Scenario 10 \\
\hline PZ & MPCA & KL & MPCA & KL & MPCA & KL & MPCA & KL & MPCA & KL \\
\hline $\mathbf{1}$ & $79 \%$ & $91 \%$ & $79 \%$ & $91 \%$ & $83 \%$ & $91 \%$ & $44 \%$ & $71 \%$ & $62 \%$ & $89 \%$ \\
\hline $\mathbf{2}$ & $77 \%$ & $91 \%$ & $77 \%$ & $91 \%$ & $86 \%$ & $91 \%$ & $47 \%$ & $71 \%$ & $60 \%$ & $89 \%$ \\
\hline $\mathbf{3}$ & $25 \%$ & $88 \%$ & $25 \%$ & $88 \%$ & $19 \%$ & $88 \%$ & $84 \%$ & $92 \%$ & $85 \%$ & $87 \%$ \\
\hline $\mathbf{4}$ & $62 \%$ & $89 \%$ & $67 \%$ & $89 \%$ & $76 \%$ & $89 \%$ & $55 \%$ & $76 \%$ & - & $88 \%$ \\
\hline $\mathbf{5}$ & $86 \%$ & $90 \%$ & $90 \%$ & $90 \%$ & $86 \%$ & $90 \%$ & $47 \%$ & $70 \%$ & $60 \%$ & $89 \%$ \\
\hline $\mathbf{6}$ & $63 \%$ & $89 \%$ & $67 \%$ & $89 \%$ & $77 \%$ & $89 \%$ & $66 \%$ & $80 \%$ & $55 \%$ & $88 \%$ \\
\hline $\mathbf{7}$ & $77 \%$ & $90 \%$ & $77 \%$ & $90 \%$ & $86 \%$ & $90 \%$ & $47 \%$ & $70 \%$ & $60 \%$ & $89 \%$ \\
\hline $\mathbf{8}$ & $76 \%$ & $89 \%$ & $76 \%$ & $89 \%$ & $81 \%$ & $89 \%$ & $47 \%$ & $70 \%$ & $60 \%$ & $88 \%$ \\
\hline $\mathbf{9}$ & $72 \%$ & $89 \%$ & $77 \%$ & $89 \%$ & $82 \%$ & $89 \%$ & $47 \%$ & $70 \%$ & $60 \%$ & $88 \%$ \\
\hline $\mathbf{1 0}$ & $61 \%$ & $91 \%$ & $75 \%$ & $91 \%$ & $71 \%$ & $91 \%$ & $47 \%$ & $71 \%$ & $64 \%$ & $89 \%$ \\
\hline
\end{tabular}

